\title{
TITLE:
}

\section{Onset of thermal convection and its flow patterns in a rectangular cavity}

\author{
$\operatorname{AUTHOR}(\mathrm{S})$ :
}

Fukazawa, Yoshinari; Funakoshi, Mitsuaki

\section{CITATION:}

Fukazawa, Yoshinari ...[et al]. Onset of thermal convection and its flow patterns in a rectangular cavity. Fluid Dynamics Research 2015, 47(6): 65505.

\section{ISSUE DATE:}

2015-10-19

URL:

http://hdl.handle.net/2433/207645

\section{RIGHT:}

This is an author-created, un-copyedited version of an article accepted for publication in 'Fluid dynamics research'. The publisher is not responsible for any errors or omissions in this version of the manuscript or any version derived from it. The Version of Record is available online at http://dx.doi.org/10.1088/0169-5983/47/6/065505.; The full-text file will be made open to the public on 19 October 2016 in accordance with publisher's 'Terms and Conditions for Self-Archiving'., この論文は出版社版でありません。引用の際には出版社版をご確認ご利用ください。; This is not the published version. Please cite only the published version. 


\title{
Onset of thermal convection and its flow patterns in a rectangular cavity
}

\author{
Yoshinari Fukazawał and Mitsuaki Funakoshi $\S$ \\ Department of Applied Analysis and Complex Dynamical Systems, Graduate School \\ of Informatics, Kyoto University, Kyoto 606-8501, Japan \\ E-mail: mitsu@acs.i.kyoto-u.ac.jp
}

\begin{abstract}
Onset of thermal convection of a fluid in a rectangular cavity of aspect ratios $A_{x}$ and $A_{y}$ is examined under the assumption that its all walls are rigid and of perfect thermal conductance exposed to a vertically linear temperature field. For several $A_{x}$ and $A_{y}$ smaller than 6 , critical Rayleigh number $R_{c}$ and the steady flow patterns of most unstable modes are computed by a Galerkin spectral method of high accuracy. Characteristics of flow patterns are examined by using upper-wall flow patterns based on near-wall velocity fields, distributions of vertical velocity, and trajectories of fluid particles. We find that a symmetry mode that is not the most unstable when the difference between $A_{x}$ and $A_{y}$ is large is the most unstable if both $A_{x}$ and $A_{y}$ are around 4 or around 5.5. The flow pattern of this mode is consistent with the results in a preceding experimental study. $R_{c}$ increases rapidly as $A_{x}$ or $A_{y}$ decreases to zero, and decreases slowly as they increase. The validity of assumption of finite roll is found to be limited even if the difference between $A_{x}$ and $A_{y}$ is large. The flow pattern for $A_{x}=A_{y}=A$ becomes more complicated and the number of convection cells increases as $A$ increases. The motion of fluid particles in each cell is roughly the circulation along closed curves around an axis that is on the central horizontal plane but is not necessarily parallel to side walls. Moreover, the motion of fluid particles near side walls can be much different from that in the central part of cavity.
\end{abstract}

$\ddagger$ Present address: Hyogo Prefectural Sanda Shounkan Senior High School, Hyogo 669-1337, Japan

$\S$ Corresponding author: mitsu@acs.i.kyoto-u.ac.jp 


\section{Introduction}

As one of the important subjects of thermal convection, the onset of convective motion in a closed cavity has been studied experimentally, theoretically, or numerically by several researchers for many years.

In 1960's and 1970's, a few important studies on the onset of thermal convection in a rectangular cavity of rigid walls were performed. Davis (1967) numerically examined the onset of thermal convection in a cavity with walls of perfect thermal conductance. If the temperatures on upper and lower walls are constants, and those on side walls are linear function of vertical coordinate connecting the temperatures on upper and lower walls, the motionless state (thermal conduction state) of a fluid in the cavity is possible. Davis considered this case, and examined the critical Rayleigh number $R_{c}$ and the flow pattern of most unstable mode that is destabilized at this Rayleigh number for several values of aspect ratios $A_{x}=L_{x} / d$ and $A_{y}=L_{y} / d$, where $L_{x}$ and $L_{y}$ are horizontal lengths of the cavity, and $d$ is its height. He computed the values of $R_{c}$ and most unstable modes under the assumption of finite roll, in which a horizontal component of fluid velocity normal to a side wall is zero. He obtained the following results: $R_{c}$ decreases with the increase in aspect ratios. The change of most unstable modes frequently occurs with the variation of aspect ratios. If both $A_{x}$ and $A_{y}$ are larger than 1 , the flow pattern of most unstable mode is a linear arrangement of several convection rolls of nearly square cross-section whose axes are horizontal and parallel to shorter side walls, as was remarked by Koschmieder (1966) in his experimental study for large aspect ratios. Davis (1967) also produced a figure (Fig. 13 of his paper) in which the dependences of $R_{c}$ and most unstable modes on aspect ratios are shown for $1 / 4 \leq A_{x}, A_{y} \leq 6$. However, the assumption of finite roll cannot be satisfied by the solutions to governing equations of thermal convection, as was pointed out Davies-Jones (1970). Catton (1970) also obtained similar results in his numerical study of the same problem, in which velocity field is assumed to be a superposition of finite rolls of different zero components.

Stork and Müller (1972) experimentally examined the onset of thermal convection in a rectangular cavity for aspect ratios $2 \leq A_{x} \leq 6$ and $0.5 \leq A_{y} \leq 6$. Their results on $R_{c}$ and the flow pattern that appears at Rayleigh number $R$ just above $R_{c}$ were compared with the results by Davis (1967) and Catton (1970), although the side walls in their experiments were not of perfect thermal conductance. Most of the above results by Davis were confirmed experimentally by Stork and Müller. However, there were a few discrepancies between the experimental and numerical results. The most remarkable discrepancy is that when both $A_{x}$ and $A_{y}$ are large and close to each other, Stork and Müller observed symmetric cell patterns rather than roll patterns corresponding to a linear arrangement of convection rolls, in the visualization of flow field near an upper wall with aluminum flakes.

After the studies by Davis (1967) and Catton (1970), there were several numerical studies aiming at the determination of $R_{c}$ and/or most unstable modes for many values 
of aspect ratios of a rectangular or cubic cavity with side walls of perfect thermal conductance (see for example, Gershuni and Zhukhovitskii (1972), Kirchartz and Oertel (1988) and Bousset et al (2008) ). Some of such studies as well as experimental studies related to the flow patterns for $R$ just above $R_{c}$ performed before 1990 were excellently reviewed in detail by Koschmieder (1993).

The computation of $R_{c}$ and most unstable modes with high accuracy was carried out by Mizushima and Matsuda (1997) for a cubic and rectangular cavities using both a Galerkin's method and a collocation method without using the assumption of finite roll. They found that $R_{c}$ for a cubic cavity is 6798 , and showed that the flow field of most unstable mode for this cavity is roughly expressed by a set of concentric spheres along which fluid flows, and is not nearly two-dimensional except for a small central region. A few results for a rectangular cavity were also obtained in this study. That is, if $A_{x}$ is increased from 1 with $A_{y}$ fixed to 1 , the change of most unstable mode between the modes of different symmetry is observed, and $R_{c}$ tends to a value close to 3000. Moreover, as both $A_{x}$ and $A_{y}$ increase, $R_{c}$ tends to 1707.76 , the value for an infinitely extended fluid layer. A little more results for a rectangular cavity were shown in Mizushima and Matsuda (1996). For integer values of $A_{x}=A_{y}$ between 1 and 6 , they computed the values of $R$ at which a mode is neutrally stable, for a few (but not all) modes of different symmetry. Puigjaner et al. (2008) examined the onset of thermal convection and bifurcations of steady convection solutions associated with the variation of $R$ for a cubic cavity. In the study with a Galerkin spectral method, they obtained $R_{c}=6799$, which is close to the value obtained by Mizushima and Matsuda (1997).

Leong et al. (1998) experimentally examined the onset of thermal convection for a cubic cavity with side walls of almost perfect thermal conductance. The $R_{c}$ obtained in this study is $6969 \pm 144$ and $7058 \pm 119$ for two cases of temperature difference between upper and lower walls. This experimental result seems consistent with the numerical result of 6798 because the deviation of average of these two central values of $R_{c}$ from 6798 is $3 \%$.

In the above preceding numerical studies, the dependence of $R_{c}$ and most unstable mode on aspect ratios $A_{x}$ and $A_{y}$ of a rectangular cavity with side walls of perfect thermal conductance was not examined systematically for several $A_{x}$ and $A_{y}$ with high accuracy. Therefore, in the present study, we aim at obtaining the values of $R_{c}$ and the detailed flow patterns of most unstable mode in such a cavity for several values of $A_{x}$ and $A_{y}$ less than 6 using a numerical method of high accuracy. Moreover, comparisons of our numerical results with experimental results in preceding relevant studies are also aimed at.

After the description of formulation of considered problem in section 2, section 3 is devoted to the explanation of numerical method used to examine the linear stability of motionless state. The convergence of numerical solutions is examined in section 4 . Numerical results on $R_{c}$ and most unstable modes are shown in section 5 . Finally, section 6 is devoted to discussion and conclusions. 


\section{Formulation}

We consider the onset of thermal convection of an incompressible viscous fluid in a rectangular cavity exposed to a vertically linear temperature field. Cartesian coordinates $\left(x^{*}, y^{*}, z^{*}\right)$ are defined in which $x^{*}$ and $y^{*}$ axes are horizontal and parallel to the side walls of cavity, $z^{*}$ axis is vertically upward, and the origin is at the center of cavity. Two aspect ratios of the cavity are defined as $A_{x} \equiv L_{x} / d$ and $A_{y} \equiv L_{y} / d$, where $L_{x}$, $L_{y}$ and $d$ respectively denote the length of cavity in the $x^{*}, y^{*}$ and $z^{*}$ directions. We assume that the external temperature field $T_{\mathrm{ex}}^{*}$ decreases linearly in upward direction and is expressed as

$$
T_{\mathrm{ex}}^{*}\left(z^{*}\right)=-\beta z^{*}+T_{0}^{*},
$$

where $\beta(>0)$ is the gradient of external temperature, and $T_{0}^{*}$ is the external temperature at $z^{*}=0$. All the walls of cavity are assumed to be rigid and of perfect thermal conductance.

Under the Boussinesq approximation, the equation of continuity, Navier-Stokes equation and energy equation with respect to velocity $\boldsymbol{v}^{*}$, temperature $T^{*}$, and pressure $p^{*}$ of fluid, are written as

$$
\begin{aligned}
& \nabla^{*} \cdot \boldsymbol{v}^{*}=0 \\
& \rho_{0}\left(\frac{\partial}{\partial t^{*}}+\boldsymbol{v}^{*} \cdot \nabla^{*}\right) \boldsymbol{v}^{*}=-\nabla^{*} p^{*}+\rho_{0} \nu \Delta^{*} \boldsymbol{v}^{*}-\rho_{0} g\left\{1-\alpha\left(T^{*}-T_{0}^{*}\right)\right\} \boldsymbol{e}_{z}, \\
& \left(\frac{\partial}{\partial t^{*}}+\boldsymbol{v}^{*} \cdot \nabla^{*}\right) T^{*}=\kappa \Delta^{*} T^{*},
\end{aligned}
$$

where

$$
\nabla^{*} \equiv \boldsymbol{e}_{x} \frac{\partial}{\partial x^{*}}+\boldsymbol{e}_{y} \frac{\partial}{\partial y^{*}}+\boldsymbol{e}_{z} \frac{\partial}{\partial z^{*}}, \quad \Delta^{*} \equiv \frac{\partial^{2}}{\partial x^{* 2}}+\frac{\partial^{2}}{\partial y^{* 2}}+\frac{\partial^{2}}{\partial z^{* 2}},
$$

and $\boldsymbol{e}_{x}, \boldsymbol{e}_{y}$ and $\boldsymbol{e}_{z}$ respectively represent the unit vectors in the $x^{*}, y^{*}$ and $z^{*}$ directions. $t^{*}$ is the time and $\rho_{0}$ is the density of fluid for $T^{*}=T_{0}^{*}$. Also $\nu, \alpha$, and $\kappa$ respectively denote the kinematic viscosity, the coefficient of thermal expansion, and the thermal diffusivity of fluid, and $g$ is the acceleration due to gravity. Boundary conditions for the velocity and temperature of fluid on the walls of cavity are given by

$$
\left\{\begin{array}{llll}
\boldsymbol{v}^{*}=0, T^{*}=T_{\mathrm{ex}}^{*}\left(z^{*}\right) & \text { on } & x^{*}= \pm \frac{L_{x}}{2}, \\
\boldsymbol{v}^{*}=0, T^{*}=T_{\mathrm{ex}}^{*}\left(z^{*}\right) & \text { on } & y^{*}= \pm \frac{L_{y}}{2}, \\
\boldsymbol{v}^{*}=0, T^{*}=T_{\mathrm{ex}}^{*}\left(z^{*}\right) & \text { on } & z^{*}= \pm \frac{d}{2} .
\end{array}\right.
$$

Here we define non-dimensionalized variables as

$$
x \equiv \frac{x^{*}}{d}, y \equiv \frac{y^{*}}{d}, z \equiv \frac{z^{*}}{d}, t \equiv \frac{\kappa}{d^{2}} t^{*}, \boldsymbol{v} \equiv \frac{d}{\kappa} \boldsymbol{v}^{*}, T \equiv \frac{T^{*}}{\beta d}, p \equiv \frac{d^{2}}{\rho_{0} \kappa \nu} p^{*} .
$$

Non-dimensionalized governing equations obtained from eqs. (2)-(4) and (6) are written as

$$
\nabla \cdot \boldsymbol{v}=0
$$




$$
\begin{aligned}
& \frac{1}{P}\left(\frac{\partial}{\partial t}+\boldsymbol{v} \cdot \nabla\right) \boldsymbol{v}=-\nabla p+\Delta \boldsymbol{v}-\left\{\frac{g d^{3}}{\kappa \nu}-R\left(T-T_{0}\right)\right\} \boldsymbol{e}_{z}, \\
& \left(\frac{\partial}{\partial t}+\boldsymbol{v} \cdot \nabla\right) T=\Delta T
\end{aligned}
$$

where

$$
\nabla \equiv \boldsymbol{e}_{x} \frac{\partial}{\partial x}+\boldsymbol{e}_{y} \frac{\partial}{\partial y}+\boldsymbol{e}_{z} \frac{\partial}{\partial z}, \quad \Delta \equiv \frac{\partial^{2}}{\partial x^{2}}+\frac{\partial^{2}}{\partial y^{2}}+\frac{\partial^{2}}{\partial z^{2}},
$$

and $T_{0}=T_{0}^{*} /(\beta d)$. Two non-dimensional parameters $R$ and $P$ in eq. (8) respectively denote the Rayleigh number and the Prandtl number defined by

$$
R \equiv \frac{\alpha g \beta d^{4}}{\kappa \nu}, \quad P \equiv \frac{\nu}{\kappa} .
$$

Non-dimensionalized boundary conditions obtained from eq. (5) are expressed as

$$
\left\{\begin{array}{lll}
\boldsymbol{v}=0, T=T_{\mathrm{ex}}(z) & \text { on } & x= \pm \frac{A_{x}}{2} \\
\boldsymbol{v}=0, T=T_{\mathrm{ex}}(z) & \text { on } & y= \pm \frac{A_{y}}{2} \\
\boldsymbol{v}=0, T=T_{\mathrm{ex}}(z) & \text { on } & z= \pm \frac{1}{2}
\end{array}\right.
$$

where

$$
T_{\mathrm{ex}}(z)=-z+T_{0}
$$

from eqs. (1) and (6).

Governing equations (7)-(9) with boundary conditions (10) have the solution of motionless state. Fluid velocity $\boldsymbol{v}_{s}$, temperature $T_{s}$, and pressure $p_{s}$ of this solution are given by

$$
\left\{\begin{array}{l}
\boldsymbol{v}_{s}=0, \\
T_{s}=T_{\mathrm{ex}}(z), \\
p_{s}=-\frac{1}{2} R z^{2}-\frac{g d^{3}}{\kappa \nu} z+p_{0},
\end{array}\right.
$$

where $p_{0}$ is the pressure at $z=0$.

If we define the deviations of velocity, temperature, and pressure from those in the motionless state as

$$
\left\{\begin{aligned}
\boldsymbol{u} & \equiv \boldsymbol{v}-\boldsymbol{v}_{s} \\
\theta & \equiv T-T_{s} \\
q & \equiv p-p_{s}
\end{aligned}\right.
$$

we obtain the following governing equations with respect to $\boldsymbol{u}, \theta$, and $q$ :

$$
\begin{aligned}
& \nabla \cdot \boldsymbol{u}=0 \\
& \frac{1}{P}\left(\frac{\partial}{\partial t}+\boldsymbol{u} \cdot \nabla\right) \boldsymbol{u}=-\nabla q+\Delta \boldsymbol{u}+R \theta \boldsymbol{e}_{z} \\
& \left(\frac{\partial}{\partial t}+\boldsymbol{u} \cdot \nabla\right) \theta=\Delta \theta+\boldsymbol{u} \cdot \boldsymbol{e}_{z}
\end{aligned}
$$


from eqs. (7)-(9), (12) and (13). Boundary conditions for $\boldsymbol{u}$ and $\theta$ obtained from eqs. (10), (12) and (13) are written as

$$
\left\{\begin{array}{lll}
\boldsymbol{u}=0, \theta=0 & \text { on } & x= \pm \frac{A_{x}}{2} \\
\boldsymbol{u}=0, \theta=0 & \text { on } & y= \pm \frac{A_{y}}{2} \\
\boldsymbol{u}=0, \theta=0 & \text { on } & z= \pm \frac{1}{2}
\end{array}\right.
$$

\section{Stability analysis of motionless state}

We examine the linear stability of motionless state on the basis of linearized version of eqs. (14)-(16) and eq. (17). The variables $\boldsymbol{u}, \theta$, and $q$ in these equations are assumed to be of the following time dependences:

$$
\left\{\begin{array}{l}
\boldsymbol{u}(x, y, z, t)=e^{\lambda t} \hat{\boldsymbol{u}}(x, y, z)+\text { c.c. }, \\
\theta(x, y, z, t)=e^{\lambda t} \hat{\theta}(x, y, z)+\text { c.c. } \\
q(x, y, z, t)=e^{\lambda t} \hat{q}(x, y, z)+\text { c.c. }
\end{array}\right.
$$

where $\hat{\boldsymbol{u}}, \hat{\theta}$ and $\hat{q}$ are complex-valued functions of $x, y$ and $z$. Also $\lambda$ is a complex constant, and c.c. denotes the complex conjugate of preceding terms. Critical Rayleigh number $R_{c}$ is defined as the value of $R$ for which the motionless state is neutrally stable, that is, the real part of $\lambda$ equals to zero. Sherman and Ostrach (1966) obtained a result implying that $\lambda$ is always real for positive $R$ if the linearized version of eqs. (14)-(16) and boundary condition (17) are satisfied. Therefore, the value of $R_{c}$ is obtained under the condition of $\lambda=0$.

Governing equations with respect to $\hat{\boldsymbol{u}}, \hat{\theta}$ and $\hat{q}$ of neutrally stable disturbances derived from the linearized version of eqs. (14)-(16) with $R=R_{c}$ and eq. (18) with $\lambda=0$ are expressed as

$$
\begin{aligned}
& \nabla \cdot \hat{\boldsymbol{u}}=0, \\
& -\nabla \hat{q}+\Delta \hat{\boldsymbol{u}}+R_{c} \hat{\theta} \boldsymbol{e}_{z}=0, \\
& \Delta \hat{\theta}+\hat{\boldsymbol{u}} \cdot \boldsymbol{e}_{z}=0
\end{aligned}
$$

Here $\hat{\boldsymbol{u}}, \hat{\theta}$ and $\hat{q}$ can be assumed to be real functions. Moreover, from eqs. (17) and (18), the following boundary conditions for $\hat{\boldsymbol{u}}$ and $\hat{\theta}$ of neutrally stable disturbances are obtained:

$$
\left\{\begin{array}{lll}
\hat{\boldsymbol{u}}=0, \hat{\theta}=0 & \text { on } & x= \pm \frac{A_{x}}{2} \\
\hat{\boldsymbol{u}}=0, \hat{\theta}=0 & \text { on } & y= \pm \frac{A_{y}}{2} \\
\hat{\boldsymbol{u}}=0, \hat{\theta}=0 & \text { on } & z= \pm \frac{1}{2}
\end{array}\right.
$$

We use a Galerkin spectral method to solve numerically eqs. (19)-(21) under boundary condition (22). We expand $\hat{\boldsymbol{u}}$ and $\hat{\theta}$ as

$$
\hat{\boldsymbol{u}}(x, y, z)=\sum_{\ell=0}^{2 L+1} \sum_{m=0}^{2 M+1} \sum_{n=0}^{2 N+1} \sum_{J=1}^{3} a_{(\ell, m, n)}^{(J)} \boldsymbol{f}_{(\ell, m, n)}^{(J)}(x, y, z),
$$




$$
\hat{\theta}(x, y, z)=\sum_{\ell=0}^{2 L+1} \sum_{m=0}^{2 M+1} \sum_{n=0}^{2 N+1} b_{(\ell, m, n)} f_{(\ell, m, n)}(x, y, z),
$$

where basis functions $\boldsymbol{f}_{(\ell, m, n)}^{(J)}(x, y, z)$ and $f_{(\ell, m, n)}(x, y, z)$ are defined by

$$
\left\{\begin{array}{l}
\boldsymbol{f}_{(\ell, m, n)}^{(1)}(x, y, z) \equiv \nabla \times F_{\ell}\left(\frac{2}{A_{x}} x\right) G_{m}\left(\frac{2}{A_{y}} y\right) G_{n}(2 z) \boldsymbol{e}_{x} \\
\boldsymbol{f}_{(\ell, m, n)}^{(2)}(x, y, z) \equiv \nabla \times G_{\ell}\left(\frac{2}{A_{x}} x\right) F_{m}\left(\frac{2}{A_{y}} y\right) G_{n}(2 z) \boldsymbol{e}_{y} \\
\boldsymbol{f}_{(\ell, m, n)}^{(3)}(x, y, z) \equiv \nabla \times G_{\ell}\left(\frac{2}{A_{x}} x\right) G_{m}\left(\frac{2}{A_{y}} y\right) F_{n}(2 z) \boldsymbol{e}_{z} \\
f_{(\ell, m, n)}(x, y, z) \equiv F_{\ell}\left(\frac{2}{A_{x}} x\right) F_{m}\left(\frac{2}{A_{y}} y\right) F_{n}(2 z)
\end{array}\right.
$$

for $\ell=0,1, \ldots, 2 L+1, m=0,1, \ldots, 2 M+1$ and $n=0,1, \ldots, 2 N+1$. Therefore, eq. (19) is satisfied for any values of expansion coefficients $a_{(\ell, m, n)}^{(J)}$. In the above basis functions, similarly to the study by Mizushima and Matsuda (1996, 1997), modified Chebyshev polynomials $F_{\ell}(\xi)$ and $G_{\ell}(\xi)$ defined by

$$
F_{\ell}(\xi) \equiv\left(1-\xi^{2}\right) T_{\ell}(\xi), \quad G_{\ell}(\xi) \equiv\left(1-\xi^{2}\right)^{2} T_{\ell}(\xi), \quad(\ell=0,1,2, \ldots),
$$

are used, where $T_{\ell}(\xi)$ is the Chebyshev polynomial of the $\ell$-th order, and $\xi=$ $2 x / A_{x}, 2 y / A_{y}$ or $2 z$. Since relations

$$
F_{\ell}( \pm 1)=G_{\ell}( \pm 1)=\left.\frac{\mathrm{d} G_{\ell}(\xi)}{\mathrm{d} \xi}\right|_{\xi= \pm 1}=0 \quad(\ell=0,1,2, \ldots)
$$

hold, boundary condition (22) is satisfied for any values of expansion coefficients $a_{(\ell, m, n)}^{(J)}$ and $b_{(\ell, m, n)}$. Both expansion (23) of $\hat{\boldsymbol{u}}$ and expansion (24) of $\hat{\theta}$ are truncated at $\ell=2 L+1, m=2 M+1$ and $n=2 N+1$ in the $x, y$ and $z$ directions, respectively.

It is known that any divergence-free vector fields in a three-dimensional simply connected space can be expressed by using two scalar potential functions. However, when we consider the velocity field in a rectangular cavity, it is difficult to express all the boundary conditions on its walls in decoupled forms of these functions. Therefore, in the present study, similarly to the preceding studies by Kessler (1987), Puigjaner et al. (2004) and Puigjaner et al. (2008), we express this velocity field by using three scalar potential functions, but with basis functions different from those in these studies. That is, eq. (23) can be rewritten as

$$
\hat{\boldsymbol{u}}(x, y, z)=\nabla \times\left[\phi_{1}(x, y, z) \boldsymbol{e}_{x}+\phi_{2}(x, y, z) \boldsymbol{e}_{y}+\phi_{3}(x, y, z) \boldsymbol{e}_{z}\right],
$$

where three scalar potential functions $\phi_{1}, \phi_{2}$, and $\phi_{3}$ are expanded as

$$
\left\{\begin{array}{l}
\phi_{1}(x, y, z)=\sum_{\ell=0}^{2 L+1} \sum_{m=0}^{2 M+1} \sum_{n=0}^{2 N+1} a_{(\ell, m, n)}^{(1)} F_{\ell}\left(\frac{2}{A_{x}} x\right) G_{m}\left(\frac{2}{A_{y}} y\right) G_{n}(2 z), \\
\phi_{2}(x, y, z)=\sum_{\ell=0}^{2 L+1} \sum_{m=0}^{2 M+1} \sum_{n=0}^{2 N+1} a_{(\ell, m, n)}^{(2)} G_{\ell}\left(\frac{2}{A_{x}} x\right) F_{m}\left(\frac{2}{A_{y}} y\right) G_{n}(2 z), \\
\phi_{3}(x, y, z)=\sum_{\ell=0}^{2 L+1} \sum_{m=0}^{2 M+1} \sum_{n=0}^{2 N+1} a_{(\ell, m, n)}^{(3)} G_{\ell}\left(\frac{2}{A_{x}} x\right) G_{m}\left(\frac{2}{A_{y}} y\right) F_{n}(2 z) .
\end{array}\right.
$$


As was explained by Puigjaner et al. (2004), by introducing these three scalar potential functions, we can impose the following decoupled boundary conditions for them on all the walls, without a loss of generality:

$$
\begin{aligned}
\phi_{1}=\phi_{2}=\phi_{3}=\frac{\partial \phi_{1}}{\partial y}=\frac{\partial \phi_{1}}{\partial z} & =\frac{\partial \phi_{2}}{\partial z}=\frac{\partial \phi_{2}}{\partial x}=\frac{\partial \phi_{3}}{\partial x}=\frac{\partial \phi_{3}}{\partial y}=0 \\
\text { on } x & = \pm \frac{A_{x}}{2}, y= \pm \frac{A_{y}}{2}, \text { or } z= \pm \frac{1}{2} .
\end{aligned}
$$

Therefore, by using basis functions satisfying boundary condition (28) in expansion (27), we can calculate the velocity field without any more consideration of boundary conditions.

Here we define the inner product $\langle\langle\boldsymbol{f}, \boldsymbol{g}\rangle\rangle$ of vector-valued functions $\boldsymbol{f}(x, y, z)$ and $\boldsymbol{g}(x, y, z)$ by

$$
\langle\langle\boldsymbol{f}, \boldsymbol{g}\rangle\rangle \equiv \int_{-\frac{A_{x}}{2}}^{\frac{A_{x}}{2}} \mathrm{~d} x \int_{-\frac{A_{y}}{2}}^{\frac{A_{y}}{2}} \mathrm{~d} y \int_{-\frac{1}{2}}^{\frac{1}{2}} \mathrm{~d} z \boldsymbol{f}(x, y, z) \cdot \boldsymbol{g}(x, y, z),
$$

and the inner product $\langle f, g\rangle$ of scalar functions $f(x, y, z)$ and $g(x, y, z)$ by

$$
\langle f, g\rangle \equiv \int_{-\frac{A_{x}}{2}}^{\frac{A_{x}}{2}} \mathrm{~d} x \int_{-\frac{A_{y}}{2}}^{\frac{A_{y}}{2}} \mathrm{~d} y \int_{-\frac{1}{2}}^{\frac{1}{2}} \mathrm{~d} z f(x, y, z) g(x, y, z) .
$$

Moreover, we introduce functions $\tilde{\boldsymbol{f}}_{(i, j, k)}^{(I)}(I=1,2,3)$ and $\tilde{f}_{(i, j, k)}$ for $i=0,1, \ldots, 2 L+$ $1, j=0,1, \ldots, 2 M+1$ and $k=0,1, \ldots, 2 N+1$ defined by

$$
\begin{aligned}
& \tilde{\boldsymbol{f}}_{(i, j, k)}^{(I)}(x, y, z) \equiv \begin{cases}\nabla \times \tilde{F}_{i}\left(\frac{2}{A_{x}} x\right) \tilde{G}_{j}\left(\frac{2}{A_{y}} y\right) \tilde{G}_{k}(2 z) \boldsymbol{e}_{x} & (\text { for } I=1), \\
\nabla \times \tilde{G}_{i}\left(\frac{2}{A_{x}} x\right) \tilde{F}_{j}\left(\frac{2}{A_{y}} y\right) \tilde{G}_{k}(2 z) \boldsymbol{e}_{y} & (\text { for } I=2), \\
\nabla \times \tilde{G}_{i}\left(\frac{2}{A_{x}} x\right) \tilde{G}_{j}\left(\frac{2}{A_{y}} y\right) \tilde{F}_{k}(2 z) \boldsymbol{e}_{z} & (\text { for } I=3),\end{cases} \\
& \tilde{f}_{(i, j, k)}(x, y, z) \equiv \tilde{F}_{i}\left(\frac{2}{A_{x}} x\right) \tilde{F}_{j}\left(\frac{2}{A_{y}} y\right) \tilde{F}_{k}(2 z),
\end{aligned}
$$

where functions $\tilde{F}_{i}(\xi)$ and $\tilde{G}_{i}(\xi)$ are defined as

$$
\tilde{F}_{i}(\xi) \equiv \frac{F_{i}(\xi)}{\sqrt{1-\xi^{2}}}, \quad \tilde{G}_{i}(\xi) \equiv \frac{G_{i}(\xi)}{\sqrt{1-\xi^{2}}}, \quad(i=0,1,2, \ldots)
$$

for $\xi=2 x / A_{x}, 2 y / A_{y}$ or $2 z$.

To obtain linear algebraic equations with respect to expansion coefficients $a_{(\ell, m, n)}^{(J)}$ and $b_{(\ell, m, n)}$, we impose the following conditions that the inner product (29) of function $\tilde{\boldsymbol{f}}_{(i, j, k)}^{(I)}(x, y, z)$ and the left-hand side of eq. (20) is zero, and that the inner product (30) of function $\tilde{f}_{(i, j, k)}(x, y, z)$ and the left-hand side of eq. (21) is zero:

$$
\begin{array}{r}
\left\langle\left\langle\tilde{\boldsymbol{f}}_{(i, j, k)}^{(I)},-\nabla \hat{q}+\Delta \hat{\boldsymbol{u}}+R_{c} \hat{\theta} \boldsymbol{e}_{z}\right\rangle\right\rangle=0 \quad(\text { for } \quad I=1,2,3, i=0,1, \ldots, 2 L+1, \\
j=0,1, \ldots, 2 M+1 \text { and } k=0,1, \ldots, 2 N+1),
\end{array}
$$


Onset of thermal convection and its flow patterns in a rectangular cavity

$$
\begin{aligned}
\left\langle\tilde{f}_{(i, j, k)}, \Delta \hat{\theta}+\hat{\boldsymbol{u}} \cdot \boldsymbol{e}_{z}\right\rangle=0 \quad(\text { for } i=0,1, \ldots, 2 L+1, \\
\quad j=0,1, \ldots, 2 M+1 \text { and } k=0,1, \ldots, 2 N+1),
\end{aligned}
$$

where expansions (23) and (24) are used for $\hat{\boldsymbol{u}}$ and $\hat{\theta}$. Here the inner product of $\tilde{\boldsymbol{f}}_{(i, j, k)}^{(I)}$ and the term of pressure gradient in eq. (33) vanishes for all $I, i, j$ and $k$, because $\tilde{\boldsymbol{f}}_{(i, j, k)}^{(I)}$ satisfies the following conditions:

$$
\tilde{\boldsymbol{f}}_{(i, j, k)}^{(I)}=0 \quad \text { on the walls, } \quad \nabla \cdot \tilde{\boldsymbol{f}}_{(i, j, k)}^{(I)}=0 \quad \text { in the cavity, }
$$

for all $I, i, j$ and $k$. Moreover, the use of $\tilde{\boldsymbol{f}}_{(i, j, k)}^{(I)}$ and $\tilde{f}_{(i, j, k)}$ rather than $\boldsymbol{f}_{(i, j, k)}^{(I)}$ and $f_{(i, j, k)}$ in eqs. (33) and (34) yields relatively simple expressions of inner products with expansion coefficients because the orthogonal relation of Chebyshev polynomials can be used.

Equations (33) and (34) are reduced to a generalized eigenvalue problem expressed by

$$
A \boldsymbol{x}=\sqrt{R_{c}} B \boldsymbol{x}
$$

where eigenvector $\boldsymbol{x}$ has $N_{t} \equiv 4(2 L+2)(2 M+2)(2 N+2)$ components, and $A$ and $B$ are square matrices of dimension $N_{t}$. The $(J-1)(2 L+2)(2 M+2)(2 N+2)+\ell(2 M+$ $2)(2 N+2)+m(2 N+2)+n+1$-th component of eigenvector $\boldsymbol{x}$ expressed as $x_{(J, \ell, m, n)}$ is given by

$$
x_{(J, \ell, m, n)}=\left\{\begin{array}{l}
a_{(\ell, m, n)}^{(J)} \quad(J=1,2,3), \\
\sqrt{R_{c}} b_{(\ell, m, n)} \quad(J=4),
\end{array}\right.
$$

for $\ell=0,1, \ldots, 2 L+1, m=0,1, \ldots, 2 M+1$ and $n=0,1, \ldots, 2 N+1$. The components of matrices $A$ and $B$ in the $(I-1)(2 L+2)(2 M+2)(2 N+2)+i(2 M+2)(2 N+2)+j(2 N+2)+$ $k+1$-th row and $(J-1)(2 L+2)(2 M+2)(2 N+2)+\ell(2 M+2)(2 N+2)+m(2 N+2)+n+1$ -th column, expressed as $a_{(I, i, j, k),(J, \ell, m, n)}$ and $b_{(I, i, j, k),(J, \ell, m, n)}$ for $A$ and $B$ respectively, are written as

$$
\begin{aligned}
& a_{(I, i, j, k),(J, \ell, m, n)}= \begin{cases}\left\langle\left\langle\tilde{\boldsymbol{f}}_{(i, j, k)}^{(I)}, \Delta \boldsymbol{f}_{(\ell, m, n)}^{(J)}\right\rangle\right\rangle & (\text { for } I=1,2,3, J=1,2,3), \\
\left\langle\tilde{f}_{(i, j, k)}, \Delta f_{(\ell, m, n)}\right\rangle & (\text { for } I=J=4), \\
0 & \text { (otherwise), }\end{cases} \\
& b_{(I, i, j, k),(J, \ell, m, n)}=\left\{\begin{array}{l}
-\left\langle\left\langle\tilde{\boldsymbol{f}}_{(i, j, k)}^{(I)}, f_{(\ell, m, n)} \boldsymbol{e}_{z}\right\rangle\right\rangle \\
-\left\langle\tilde{f}_{(i, j, k)}, \boldsymbol{f}_{(\ell, m, n)}^{(J)} \cdot \boldsymbol{e}_{z}\right\rangle \\
0 \quad \text { (otherwise), }
\end{array} \quad(\text { for } I=4, J=1,2,3),\right.
\end{aligned}
$$

for $i=0,1, \ldots, 2 L+1, j=0,1, \ldots, 2 M+1, k=0,1, \ldots, 2 N+1$, and $\ell=$ $0,1, \ldots, 2 L+1, m=0,1, \ldots, 2 M+1, n=0,1, \ldots, 2 N+1$.

As was pointed out by Mizushima and Matsuda $(1996,1997)$ and by Puigjaner et al. (2004), because of the symmetry of eqs. (19)-(22) under the reflections with respect to three planes $x=0, y=0$ and $z=0$, it is sufficient to solve these equations separately for each of eight symmetry modes. These modes are expressed as $\hat{\theta}\left(s_{x}, s_{y}, s_{z}\right)$ 
mode, where $s_{x}$ (or $\left.s_{y}, s_{z}\right)=e[o]$ implies that $\hat{\theta}$ is an even [odd] function of $x$ (or $y, z$ ). Therefore, for example in $\hat{\theta}(o, o, e)$ mode, $\hat{\theta}$ is an odd function of $x$ and $y$, and also an even function of $z$. In $\hat{\theta}\left(s_{x}, s_{y}, s_{z}\right)$ mode, the symmetries of $x, y$ and $z$ components of $\hat{\boldsymbol{u}}$ are $\left(\bar{s}_{x}, s_{y}, \bar{s}_{z}\right),\left(s_{x}, \bar{s}_{y}, \bar{s}_{z}\right)$ and $\left(s_{x}, s_{y}, s_{z}\right)$, respectively, where $\bar{s}_{x}$ (or $\left.\bar{s}_{y}, \bar{s}_{z}\right)=o[e]$ if $s_{x}$ (or $\left.s_{y}, s_{z}\right)=e[o]$. It is noted that the symmetries of $\hat{\theta}$ and $z$ component of $\hat{\boldsymbol{u}}$ are the same.

In solving eq. (35) for each symmetry mode, only $N_{t} / 8$ components of $\boldsymbol{x}$ with the values of $(\ell, m, n)$ in eq. (36) corresponding to the specified symmetry in expansions (23) and (24) are required to be considered. The vector consisting of such components is called $\tilde{\boldsymbol{x}}$. Moreover, for these $N_{t} / 8$ sets of $(\ell, m, n)$, the values of $a_{(I, i, j, k),(J, \ell, m, n)}$ and $b_{(I, i, j, k),(J, \ell, m, n)}$ defined by eqs. (37) and (38) can be non-zero only for the $N_{t} / 8$ sets of $(i, j, k)$ corresponding to appropriate symmetry. Therefore, we can use matrices $\tilde{A}$ and $\tilde{B}$ of dimension $N_{t} / 8$ consisting of such components in place of $A$ and $B$ in eq. (35). We thus obtain for each symmetry mode the following equation of reduced dimension $N_{t} / 8=4(L+1)(M+1)(N+1)$ :

$$
\tilde{A} \tilde{\boldsymbol{x}}=\sqrt{R_{c}} \tilde{B} \tilde{\boldsymbol{x}}
$$

Equation (39) is rewritten as

$$
C \tilde{\boldsymbol{x}}=\frac{1}{\sqrt{R_{c}}} \tilde{\boldsymbol{x}}
$$

where $C=\tilde{A}^{-1} \tilde{B}$. The $i$-th column $\boldsymbol{c}_{i}$ of $C$ is obtained by solving simultaneous linear equation $\tilde{A} \boldsymbol{c}_{i}=\boldsymbol{b}_{i}$ for $i=1,2, \ldots, N_{t} / 8$, where $\boldsymbol{b}_{i}$ is the $i$-th column of $\tilde{B}$. We used the QR algorithm to solve eigenvalue problem (40).

The value of $R_{c}$ for $\hat{\theta}\left(s_{x}, s_{y}, s_{z}\right)$ mode, obtained from the maximum eigenvalue of eq. (40), is called $R_{c}^{\left(s_{x}, s_{y}, s_{z}\right)}$ hereinafter. Critical Rayleigh number $R_{c}$ is the minimum of $R_{c}^{\left(s_{x}, s_{y}, s_{z}\right)}$ 's for all $s_{x}, s_{y}$ and $s_{z}$, because $\hat{\boldsymbol{u}}$ and $\hat{\theta}$ are generally expressed as the superposition of the velocity and temperature fields restricted to each symmetry mode. The symmetry mode corresponding to critical Rayleigh number is called the most unstable mode hereinafter. The velocity and temperature fields of most unstable mode are obtained from the eigenvector $\tilde{\boldsymbol{x}}$ of eq. (40) for this mode associated with the eigenvalue corresponding to critical Rayleigh number.

\section{Convergence of Numerical Solutions}

The value of $R_{c}^{\left(s_{x}, s_{y}, s_{z}\right)}$ for truncation parameters $(L, M, N)$ is denoted by $R_{c}^{[L, M, N]}$. Relative variations of $R_{c}^{[L, M, N]}$ with the increase in $L, M$ and $N$ by 1 are defined by

$$
\left\{\begin{array}{c}
\varepsilon_{x}^{[L, M, N]}=\frac{\left|R_{c}^{[L+1, M, N]}-R_{c}^{[L, M, N]}\right|}{R_{c}^{[L, M, N]}}, \\
\varepsilon_{y}^{[L, M, N]}=\frac{\left|R_{c}^{[L, M+1, N]}-R_{c}^{[L, M, N]}\right|}{R_{c}^{[L, M, N]}}, \\
\varepsilon_{z}^{[L, M, N]}=\frac{\left|R_{c}^{[L, M, N+1]}-R_{c}^{[L, M, N]}\right|}{R_{c}^{[L, M, N]}},
\end{array}\right.
$$



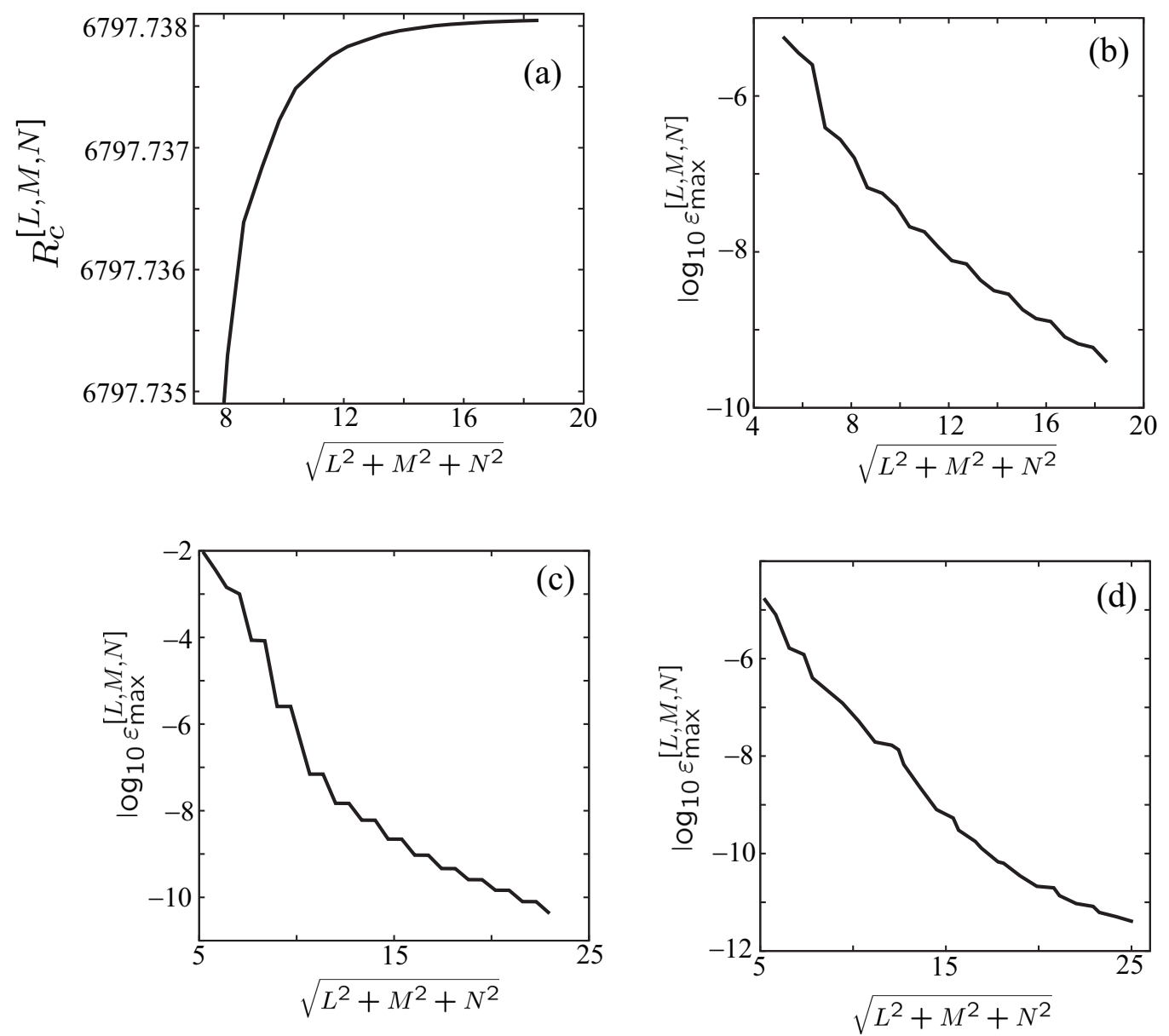

Figure 1. Examples of dependences of $R_{c}^{[L, M, N]}$ and $\varepsilon_{\max }^{[L, M, N]}$ on $\sqrt{L^{2}+M^{2}+N^{2}}$. (a) and (b): $R_{c}^{[L, M, N]}$ and $\varepsilon_{\max }^{[L, M, N]}$ for $\hat{\theta}(e, o, e) \operatorname{mode}, A_{x}=A_{y}=1$. (c): $\varepsilon_{\max }^{[L, M, N]}$ for $\hat{\theta}(o, o, e)$ mode, $A_{x}=A_{y}=6$. (d): $\varepsilon_{\max }^{[L, M, N]}$ for $\hat{\theta}(e, o, e)$ mode, $A_{x}=A_{y}=0.125$.

respectively. Using these variables, maximum relative variation $\varepsilon_{\max }^{[L, M, N]}$ at $(L, M, N)$ is expressed as

$$
\varepsilon_{\max }^{[L, M, N]}=\max \left(\varepsilon_{x}^{[L, M, N]}, \varepsilon_{y}^{[L, M, N]}, \varepsilon_{z}^{[L, M, N]}\right) .
$$

In the computation of $R_{c}^{\left(s_{x}, s_{y}, s_{z}\right)}$, we start with $L=M=N=3$ or 4 . We then repeat the procedure that $L($ or $M, N)$ is increased by 1 if $\varepsilon_{\max }^{[L, M, N]}=\varepsilon_{x}^{[L, M, N]}$ (or $\left.\varepsilon_{\max }^{[L, M, N]}=\varepsilon_{y}^{[L, M, N]}, \varepsilon_{z}^{[L, M, N]}\right)$. We observe that $\varepsilon_{\max }^{[L, M, N]}$ decreases monotonically and $R_{c}^{[L, M, N]}$ approaches a certain value, with the increase in $\sqrt{L^{2}+M^{2}+N^{2}}$ under this procedure. An example of dependences of $R_{c}^{[L, M, N]}$ and $\varepsilon_{\max }^{[L, M, N]}$ on $\sqrt{L^{2}+M^{2}+N^{2}}$ is shown in Figs. 1 (a) and (b) for $\hat{\theta}(e, o, e)$ mode, the most unstable mode, for $A_{x}=A_{y}=1$. In these figures, the tendency of $R_{c}^{[L, M, N]}$ to 6797.738 and roughly exponential decrease in $\varepsilon_{\max }^{[L, M, N]}$ with the increase in $\sqrt{L^{2}+M^{2}+N^{2}}$ are shown. Similar convergence is observed also for different aspect ratios, as illustrated in Figs. 1 (c) and (d) for the most unstable mode for $A_{x}=A_{y}=6$ and 0.125 , respectively.

In most calculations of $R_{c}^{\left(s_{x}, s_{y}, s_{z}\right)}$, the values of $(L, M, N)$ are increased until $N_{t} / 8$ 
reaches about 7000 . Because $\varepsilon_{\max }^{[L, M, N]}$ reduces to $10^{-9}$ at this largest value of $(L, M, N)$ called $\left(L_{m}, M_{m}, N_{m}\right)$, for $A_{x}$ and $A_{y}$ satisfying $0.01 \leq A_{x}, A_{y} \leq 6$, the values of $R_{c}^{\left[L_{m}, M_{m}, N_{m}\right]}$ are regarded as $R_{c}^{\left(s_{x}, s_{y}, s_{z}\right)}$. Here it is noted that the values of $\left(L_{m}, M_{m}, N_{m}\right)$ depend on aspect ratios and the symmetry of mode, probably reflecting the difference in complexity of geometrical structures of velocity and temperature fields in $x, y$ and $z$ directions.
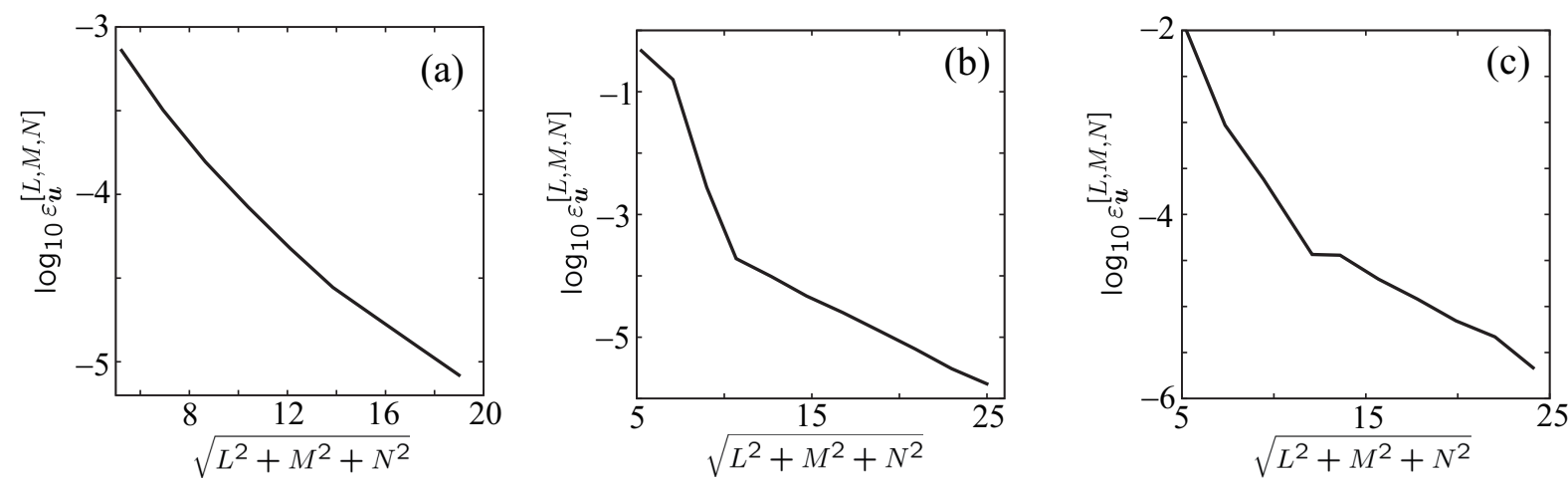

Figure 2. Examples of dependence of $\varepsilon_{\boldsymbol{u}}^{[L, M, N]}$ on $\sqrt{L^{2}+M^{2}+N^{2}}$. (a): $A_{x}=A_{y}=1$, $\hat{\theta}(e, o, e)$ mode. (b): $A_{x}=A_{y}=6, \hat{\theta}(o, o, e)$ mode. (c): $A_{x}=A_{y}=0.125, \hat{\theta}(e, o, e)$ mode.

Velocity field of $\hat{\theta}\left(s_{x}, s_{y}, s_{z}\right)$ mode denoted by $\hat{\boldsymbol{u}}^{\left(s_{x}, s_{y}, s_{z}\right)}$ is determined from eq. (23) by using expansion coefficients $a_{(\ell, m, n)}^{(J)}$ obtained from eigenvector $\tilde{\boldsymbol{x}}$ of eq. (40) for $\hat{\theta}\left(s_{x}, s_{y}, s_{z}\right)$ mode associated with the maximum eigenvalue. In order to examine the convergence of velocity fields with the increase in truncation parameters, a measure of difference in velocity fields for different truncation parameters, $\varepsilon_{\boldsymbol{u}}^{[L, M, N]}$, defined by

$$
\varepsilon_{\boldsymbol{u}}^{[L, M, N]}=\frac{\max _{x, y, z}\left|\hat{\boldsymbol{u}}^{[\tilde{L}, \tilde{M}, \tilde{N}]}(x, y, z)-\hat{\boldsymbol{u}}^{[L, M, N]}(x, y, z)\right|}{\max _{x, y, z}\left|\hat{\boldsymbol{u}}^{[L, M, N]}(x, y, z)\right|}
$$

is introduced, where $\hat{\boldsymbol{u}}^{[L, M, N]}$ is the velocity field for truncation parameters $(L, M, N)$. Also $(\tilde{L}, \tilde{M}, \tilde{N})$ are the values of truncation parameters after three repetitions of the aforementioned procedure yielding the increase in one of them by 1 from $(L, M, N)$, which implies that $\tilde{L}+\tilde{M}+\tilde{N}=L+M+N+3 . \varepsilon_{\boldsymbol{u}}^{[L, M, N]}$ decreases in an approximately exponential way as $\sqrt{L^{2}+M^{2}+N^{2}}$ increases at least if $\sqrt{L^{2}+M^{2}+N^{2}}$ is larger than 10, as illustrated in Fig. 2 for the most unstable modes for $A_{x}=A_{y}=1,6$ and 0.125 . Since the values of $\varepsilon_{\boldsymbol{u}}^{\left[L_{m}, M_{m}, N_{m}\right]}$ are as small as $10^{-5}$ or $10^{-6}$ for any $A_{x}$ and $A_{y}$ considered in the present study, the velocity field $\hat{\boldsymbol{u}}^{\left[L_{m}, M_{m}, N_{m}\right]}$ is used as $\hat{\boldsymbol{u}}^{\left(s_{x}, s_{y}, s_{z}\right)}$.

\section{Results}

5.1 Critical Rayleigh number and most unstable mode 


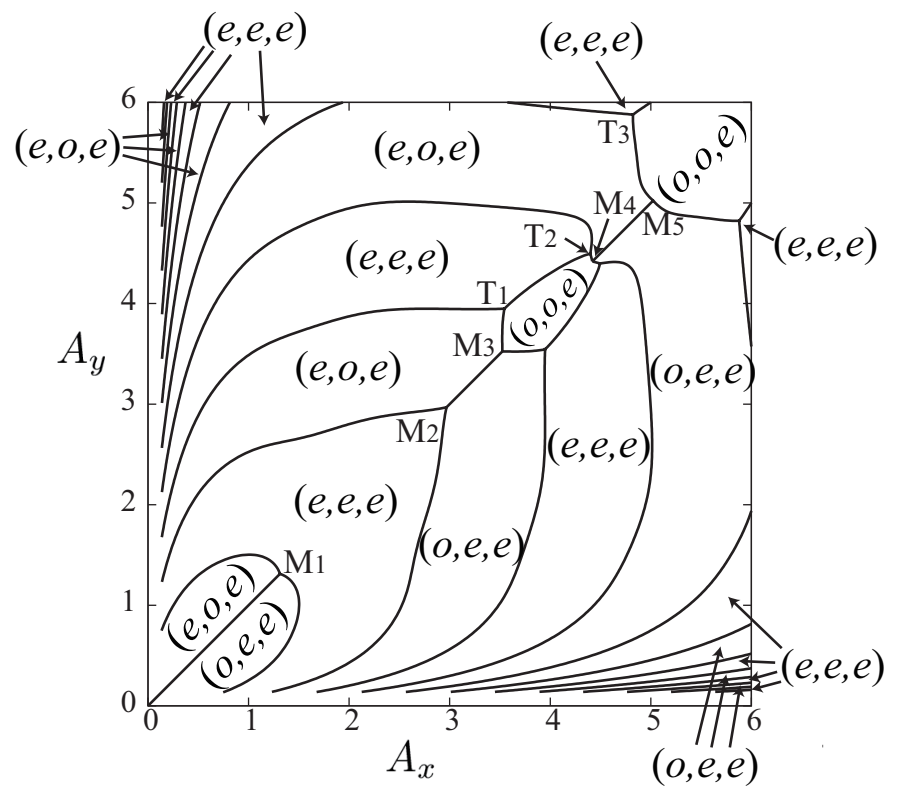

Figure 3. Symmetry in $\hat{\theta}$ of most unstable mode for $0.14 \leq A_{x}, A_{y} \leq 6$.

Most unstable modes for $A_{x}$ and $A_{y}$ within the region $0.14 \leq A_{x}, A_{y} \leq 6$ are shown in Fig. 3, where regions of each symmetry in $\hat{\theta}$ of most unstable mode are depicted. Because of the geometry of the system we consider, $R_{c}^{\left(s_{x}, s_{y}, s_{z}\right)}$ for any $\left(A_{x}, A_{y}\right)$ is the same as $R_{c}^{\left(s_{y}, s_{x}, s_{z}\right)}$ for exchanged aspect ratios $\left(A_{y}, A_{x}\right)$. Therefore, the boundary lines in Fig. 3 are symmetrical with respect to the diagonal line $A_{x}=A_{y}$. In the special case of $A_{x}=A_{y}$, if $\hat{\theta}(e, o, e)$ mode is the most unstable, $\hat{\theta}(o, e, e)$ mode is also most unstable from the relation $R_{c}^{(e, o, e)}=R_{c}^{(o, e, e)}$, which yields diagonal boundary lines in Fig. 3. The symmetry of most unstable mode is always $\hat{\theta}\left(s_{x}, s_{y}, e\right)$ in which $s_{x}$ and $s_{y}$ are either $e$ or $o$. This reflects the fact that there is vertically only one convection cell rather than two or more cells in the flow pattern of most unstable mode for any $\left(A_{x}, A_{y}\right)$. Figure 3 is similar to the result by Davis (1967) shown in Fig. 13 of his paper. That is, for fixed $A_{x}$ [or $\left.A_{y}\right]$, with the increase in $A_{y}\left[A_{x}\right]$ from a value larger than $A_{x}\left[A_{y}\right]$, we observe several changes of most unstable mode between $\hat{\theta}(e, e, e)$ and $\hat{\theta}(e, o, e)[\hat{\theta}(o, e, e)]$ modes.

However, there are two remarkable discrepancies between these figures. First, we observe two regions where $\hat{\theta}(o, o, e)$ mode is the most unstable near the diagonal line around $A_{x}=A_{y}=4$ and 5.5, unlike the Davis' result. This difference is probably due to the assumption of finite roll adopted by Davis. As far as the authors know, the existence of these regions was not shown in the preceding studies for the boundary condition considered in the present study, although Mizushima and Matsuda (1996) obtained the result that $R_{c}^{(o, o, e)}$ is smaller than $R_{c}^{(o, e, e)}\left(=R_{c}^{(e, o, e)}\right)$ for $A_{x}=A_{y}=4$ and 6 without any reference to the value of $R_{c}^{(e, e, e)}$. The existence of regions of most unstable $\hat{\theta}(o, o, e)$ mode in Fig. 3 yields many sets of aspect ratios $\left(A_{x}, A_{y}\right)$ for which critical Rayleigh numbers for three symmetry modes are the same, which correspond to the intersections of three boundary lines. In this figure, such sets with the same $A_{x}$ and $A_{y}$ are denoted 
by $\mathrm{M}_{1}\left(A_{x}=A_{y}=1.312\right), \mathrm{M}_{2}(2.968), \mathrm{M}_{3}(3.524), \mathrm{M}_{4}$ (4.418) and $\mathrm{M}_{5}$ (5.023), whereas those with different $A_{x}$ and $A_{y}$ are the sets denoted by $\mathrm{T}_{1}\left(A_{x}=3.544, A_{y}=3.948\right), \mathrm{T}_{2}$ $(4.398,4.495)$ and $\mathrm{T}_{3}(4.823,5.878)$, and the ones obtained by exchanging $A_{x}$ and $A_{y}$ of these sets.

Secondly, if $A_{x}$ [or $\left.A_{y}\right]$ is decreased to zero with $A_{y}\left[A_{x}\right]$ fixed to a value, we observe frequent changes of most unstable mode between $\hat{\theta}(e, e, e)$ and $\hat{\theta}(e, o, e)[\hat{\theta}(o, e, e)]$ modes. These changes occur more frequently for smaller $A_{x}$. That is, the lengths of $A_{x}$ intervals of each symmetry mode decrease as $A_{x}$ decreases, as shown most clearly for $A_{y}=6$ in Fig. 3. If $A_{x}$ is decreased from 3 with $A_{y}$ fixed to 3 , the changes of most unstable mode occur at $A_{x}=0.562,0.298,0.194$ and 0.138. Moreover, for $A_{y}=1$, as $A_{x}$ decreases from 1 , the changes are observed at $A_{x}=0.253,0.090,0.047,0.029,0.020 .0 .015$ and 0.011 , although most of these changes are not shown in Fig. 3. These frequent changes of most unstable mode were not shown explicitly by Davis (1967), although such behavior could be inferred from the bend of boundary lines in the region of small $A_{x}$ or small $A_{y}$ of his Fig. 13.

Table 1. $R_{c}$ and the symmetry of most unstable mode for a few typical aspect ratios. When both $\hat{\theta}(e, o, e)$ and $\hat{\theta}(o, e, e)$ modes are most unstable, the $\left(L_{m}, M_{m}, N_{m}\right)$ for $\hat{\theta}(e, o, e)$ mode is shown.

\begin{tabular}{|c|c|c|c|c|}
\hline$A_{x}$ & $A_{y}$ & $R_{c}$ & symmetry & $\left(L_{m}, M_{m}, N_{m}\right)$ \\
\hline 0.125 & 0.125 & $1.009901 \times 10^{7}$ & $\hat{\theta}(e, o, e), \hat{\theta}(o, e, e)$ & $(8,6,23)$ \\
\hline 0.125 & 1 & $6.336681 \times 10^{5}$ & $\hat{\theta}(e, e, e)$ & $(5,12,17)$ \\
\hline 0.125 & 6 & $5.873073 \times 10^{5}$ & $\hat{\theta}(e, e, e)$ & $(5,17,16)$ \\
\hline 1 & 1 & 6797.738 & $\hat{\theta}(e, o, e), \hat{\theta}(o, e, e)$ & $(12,11,11)$ \\
\hline 1 & 6 & 3030.469 & $\hat{\theta}(e, e, e)$ & $(10,13,11)$ \\
\hline 6 & 6 & 1761.788 & $\hat{\theta}(o, o, e)$ & $(18,18,4)$ \\
\hline
\end{tabular}

$R_{c}$ decreases with the increase in $A_{x}$ and $A_{y}$. This decrease is slow for large aspect ratios, whereas the increase in $R_{c}$ when either or both of $A_{x}$ and $A_{y}$ approach zero is quite rapid. These results are explained by the fact that stronger suppression of onset of convection by rigid side walls is expected for smaller aspect ratios. $R_{c}$ and the symmetry of most unstable mode for a few typical aspect ratios are shown in Table 1. For a cubic cavity of $A_{x}=A_{y}=1$, we obtain $R_{c}=6797.738$, which is very close to 6798 obtained by Mizushima and Matsuda (1997), and 6799 by Puigjaner et al. (2008). Moreover, the values of $R_{c}$ for $\left(A_{x}, A_{y}\right)=(1,6)$ and $(6,6), 3030.469$ and 1761.788 , are close to those obtained by Mizushima and Matsuda (1996), 3026.72 and 1761.75, respectively. $R_{c}=1761.788$ for $A_{x}=A_{y}=6$ is slightly larger than 1707.76 known as $R_{c}$ for a fluid between two parallel walls of infinite extension. If $A_{y}$ is increased to infinity with $A_{x}$ 
fixed to $1, R_{c}$ is expected to asymptote to the critical Rayleigh number $R_{c y}^{\infty}$ for an infinite channel of square cross section with rigid and perfectly conducting walls. Daniels and Ong (1990) obtained $R_{c y}^{\infty}=2944.28$. Our $R_{c}$ for $\left(A_{x}, A_{y}\right)=(1,6), 3030.469$, is larger than $R_{c y}^{\infty}$ by $2.9 \%$. However, since additional computation for $\left(A_{x}, A_{y}\right)=(1,9)$ gives $R_{c}=2981.68$, which is closer to $R_{c y}^{\infty}$ (deviation of $1.3 \%$ ), the above expectation is confirmed. If one of $A_{x}$ and $A_{y}$ is much smaller than 1 and the other is 1 or more, $R_{c}$ is roughly determined by the smaller aspect ratio, as illustrated in Table 1 by $R_{c}$ 's for $\left(A_{x}, A_{y}\right)=(0.125,1)$ and $(0.125,6)$. This property may reflect the strong effect of two side walls of small spacing in the onset of thermal convection. If both $A_{x}$ and $A_{y}$ are much smaller than 1 , we obtain very large value of $R_{c}$, as illustrated for $A_{x}=A_{y}=0.125$ in Table 1.

$R_{c}$ and the symmetry of most unstable mode for many more aspect ratios are shown in Table 2. Comparison of $R_{c}$ shown in Tables 1 and 2 with experimental data is not easy because critical Rayleigh number is not obtained with sufficient accuracy in experimental studies, probably owing to the difficulty in determining it. For example, the approximate values of $R_{c}$ by Stork and Müller (1972), read from the figures in their paper, are $2250-2500$ for $\left(A_{x}, A_{y}\right)=(2,2), 1900-2100$ for $(2,6), 1600-1800$ for $(4,4)$, and $1650-1850$ for $(4,6)$ and $(6,6)$. Moreover, in the experiments by Kirchartz and Oertel (1988) with almost perfectly conducting side walls, they obtained approximate values of $R_{c}=1900-2000$ for $\left(A_{x}, A_{y}\right)=(4,4)$ and $1800-1900$ for $(4,6)$. Although these results are roughly consistent with our $R_{c}$ in Tables 1 and 2 , it is difficult to compare numerical and experimental values of $R_{c}$ with high accuracy.

Table 2. $R_{c}$ and the symmetry of most unstable mode for several aspect ratios.

\begin{tabular}{|c|c|c|c||c|c|c|c|}
\hline$A_{x}$ & $A_{y}$ & $R_{c}$ & symmetry & $A_{x}$ & $A_{y}$ & $R_{c}$ & symmetry \\
\hline 0.25 & 0.25 & $6.572946 \times 10^{5}$ & $\hat{\theta}(e, o, e), \hat{\theta}(o, e, e)$ & 2 & 6 & 1927.471 & $\hat{\theta}(e, o, e)$ \\
\hline 0.5 & 0.5 & $4.941374 \times 10^{4}$ & $\hat{\theta}(e, o, e), \hat{\theta}(o, e, e)$ & 3 & 3 & 1967.826 & $\hat{\theta}(e, o, e), \hat{\theta}(o, e, e)$ \\
\hline 1 & 2 & 3756.421 & $\hat{\theta}(e, e, e)$ & 3 & 4 & 1872.318 & $\hat{\theta}(e, e, e)$ \\
\hline 1 & 3 & 3296.435 & $\hat{\theta}(e, o, e)$ & 3 & 5 & 1826.170 & $\hat{\theta}(e, o, e)$ \\
\hline 1 & 4 & 3140.486 & $\hat{\theta}(e, e, e)$ & 3 & 6 & 1799.766 & $\hat{\theta}(e, o, e)$ \\
\hline 1 & 5 & 3069.093 & $\hat{\theta}(e, o, e)$ & 4 & 4 & 1838.152 & $\hat{\theta}(o, o, e)$ \\
\hline 2 & 2 & 2317.169 & $\hat{\theta}(e, e, e)$ & 4 & 5 & 1805.161 & $\hat{\theta}(e, o, e)$ \\
\hline 2 & 3 & 2096.816 & $\hat{\theta}(e, o, e)$ & 4 & 6 & 1779.245 & $\hat{\theta}(e, e, e)$ \\
\hline 2 & 4 & 2000.226 & $\hat{\theta}(e, e, e)$ & 5 & 5 & 1789.895 & $\hat{\theta}(e, o, e), \hat{\theta}(o, e, e)$ \\
\hline 2 & 5 & 1953.589 & $\hat{\theta}(e, o, e)$ & 5 & 6 & 1772.126 & $\hat{\theta}(e, e, e)$ \\
\hline
\end{tabular}

An example of repeated changes of most unstable mode between $\hat{\theta}(e, o, e)$ and 
$\hat{\theta}(e, e, e)$ modes with the increase in $A_{y}$ for fixed $A_{x}$ is shown in Fig. 4(a). In this figure, the dependences of $R_{c}^{(e, o, e)}$ and $R_{c}^{(e, e, e)}$ on $A_{y}$ for $A_{x}=1$ are shown. Since the $R_{c}^{\left(s_{x}, s_{y}, s_{z}\right)}$ for all other symmetry modes is always larger than $R_{c}^{(e, o, e)}$ and $R_{c}^{(e, e, e)}$ for $1<A_{y} \leq 6$, $R_{c}$ is the smaller of $R_{c}^{(e, o, e)}$ and $R_{c}^{(e, e, e)}$. With the increase in $A_{y}, R_{c}$ decreases, and changes of most unstable mode occur at $A_{y}$ 's composed of $A_{y c}^{(1)}=1.503$ (not shown in this figure), $A_{y c}^{(2)}=2.525, A_{y c}^{(3)}=3.487, A_{y c}^{(4)}=4.434$ and $A_{y c}^{(5)}=5.375$. Similar changes of most unstable mode were observed also by Mizushima and Matsuda (1996). The differences between their and our $A_{y}$ 's at which changes of most unstable mode occur are less than 0.1. The difference between $R_{c}^{(e, o, e)}$ and $R_{c}^{(e, e, e)}$ decreases rapidly with the increase in $A_{y}$, as shown in Fig. 4(a). For example, the largest differences between them within the intervals of $A_{y c}^{(j)} \leq A_{y} \leq A_{y c}^{(j+1)}$ are 288, 75, 31 and 16 for $j=1,2,3$ and 4 , respectively.
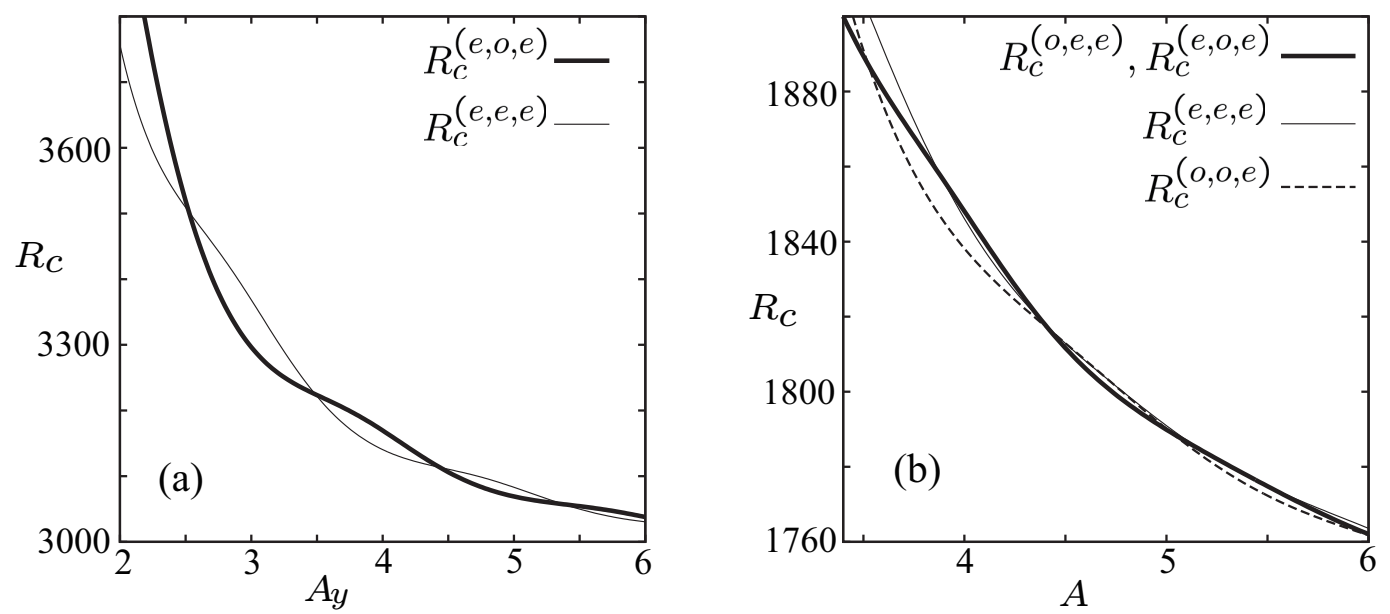

Figure 4. (a) $R_{c}^{(e, o, e)}$ and $R_{c}^{(e, e, e)}$ for $A_{x}=1$. (b) $R_{c}^{\left(s_{x}, s_{y}, e\right)}$ for four symmetry modes with $s_{x}$ and $s_{y}$ either $e$ or $o$. $A_{x}=A_{y}=A$.

For a cavity of square horizontal cross-section with $A_{x}=A_{y}=A, \hat{\theta}(e, o, e)$, $\hat{\theta}(o, e, e), \hat{\theta}(e, e, e)$ or $\hat{\theta}(o, o, e)$ mode is the most unstable depending on $A$, as shown in Fig. 3. Dependences of $R_{c}^{\left(s_{x}, s_{y}, e\right)}$ with $s_{x}$ and $s_{y}$ either $e$ or $o$ on $A$ for $3.4 \leq A \leq 6$ are shown in Fig. 4(b). Changes of most unstable mode are observed at $A=3.524,4.418$ and 5.023, which correspond to $\mathrm{M}_{3}, \mathrm{M}_{4}$ and $\mathrm{M}_{5}$ in Fig. 3. $\hat{\theta}(e, e, e)$ mode is not the most unstable for any $A$ satisfying $2.968<A \leq 6$, although it is not shown clearly in Fig. 4(b). Differences of $R_{c}^{\left(s_{x}, s_{y}, e\right)}$ for four symmetry modes shown in Fig. 4(b) again decrease rapidly with increasing $A$.

Because of the rapid decrease in difference between $R_{c}$ for the most unstable mode and $R_{c}^{\left(s_{x}, s_{y}, s_{z}\right)}$ for some other modes with the increase in $A_{x}$ and/or $A_{y}$, high accuracy in numerical computations is required to determine the most unstable mode if either or both of $A_{x}$ and $A_{y}$ are large. Moreover, it is suggested that quite careful setup of apparatus is necessary in the experiments for large aspect ratios aiming at determining the flow pattern that appears first as Rayleigh number is slowly increased, because 
small imperfection in boundary conditions may cause the appearance of a different flow pattern.

5.2 Flow patterns of most unstable mode

As was shown in the previous subsection, the most unstable mode for any $\left(A_{x}, A_{y}\right)$ satisfying $A_{x} \leq 6$ and $A_{y} \leq 6$ is $\hat{\theta}\left(s_{x}, s_{y}, e\right)$ modes in which $s_{x}$ and $s_{y}$ are either $e$ or $o$. Since the vertical component $w$ of velocity field $\hat{\boldsymbol{u}}$ of such modes is also an even function of $z$, the flow patterns of most unstable mode should be composed of odd number of convection cells in the vertical direction. In the numerical computations for $\left(A_{x}, A_{y}\right)$ satisfying $A_{x} \leq 6$ and $A_{y} \leq 6$, we find that the flow patterns of most unstable mode are composed of only one convection cell rather than three or more cells in the vertical direction. Therefore, flow patterns near the upper wall $z=1 / 2$, called upper-wall flow patterns hereinafter, are expected to give important information on the detailed structure of velocity fields of most unstable mode.

An upper-wall flow pattern is expressed by streamlines based on the $x$ and $y$ velocity components, $u_{w}(x, y)$ and $v_{w}(x, y)$, respectively, of near-wall velocity field defined in the following expansion of $x$ and $y$ components of $\hat{\boldsymbol{u}}, u$ and $v$, respectively, near the upper wall:

$$
\left\{\begin{array}{l}
u(x, y, z)=\left(\frac{1}{2}-z\right) u_{w}(x, y)+\mathrm{O}\left(\frac{1}{2}-z\right)^{2}, \\
v(x, y, z)=\left(\frac{1}{2}-z\right) v_{w}(x, y)+\mathrm{O}\left(\frac{1}{2}-z\right)^{2} .
\end{array}\right.
$$

The relation between the geometrical structure of a three-dimensional steady flow near a rigid wall and the wall flow pattern on this wall associated with this three-dimensional flow was examined in several studies such as those on separation and reattachment of flows (see for example Délery (2001) and Surana et al. (2006) and references therein). It is widely known that the intersection of streamlines of a three-dimensional flow of an incompressible fluid with a rigid wall at a non-zero angle to the wall surface is possible only at the equilibrium points of near-wall velocity field. Therefore, in the present problem, streamlines of $\hat{\boldsymbol{u}}$ that intersect the upper wall at a non-zero angle to its surface can be observed only at the equilibrium points of near-wall velocity field defined by $\left(u_{w}, v_{w}\right)$. At these equilibrium points, horizontal divergence is defined by

$$
D_{w}=\frac{\partial u_{w}}{\partial x}+\frac{\partial v_{w}}{\partial y}
$$

When $z$ component of $\hat{\boldsymbol{u}}, w$, is expanded as

$$
w(x, y, z)=\frac{1}{2}\left(\frac{1}{2}-z\right)^{2} w_{w}(x, y)+\mathrm{O}\left(\frac{1}{2}-z\right)^{3},
$$

near the upper wall, equation

$$
-D_{w}+w_{w}=0,
$$

is derived from the equation of continuity. 
If $D_{w}<0$ is satisfied at an equilibrium point, flow separation occurs at this point, and a descending flow is expected within a vertical cylindrical region around it. We call such a point a downward equilibrium point of upper-wall flow pattern. An upward equilibrium point is defined similarly by the condition of $D_{w}>0$. In vertical cylindrical regions around this equilibrium point, ascending flows are expected. There are also equilibrium points with $D_{w}=0$. We call such points neutral equilibrium points because there is no ascending or descending flow around them. Moreover, as expected from eq. (47), $\left|D_{w}\right|$ of an equilibrium point is a measure of the strength of ascending or descending flow near it. Equilibrium points of near-wall velocity field can be classified also by using two eigenvalues $\lambda_{1}$ and $\lambda_{2}$ of the Jacobian matrix

$$
J=\left(\begin{array}{ll}
\frac{\partial u_{w}}{\partial x} & \frac{\partial u_{w}}{\partial y} \\
\frac{\partial v_{w}}{\partial x} & \frac{\partial v_{w}}{\partial y}
\end{array}\right),
$$

at these points, for which $\lambda_{1}+\lambda_{2}=D_{w}$ is satisfied. That is, an equilibrium point is a node if $\lambda_{1}$ and $\lambda_{2}$ are real and of the same sign, is a saddle if they are real and of the opposite signs, and is a spiral if $\lambda_{1}$ and $\lambda_{2}=\lambda_{1}^{*}$ are complex. Here $\lambda_{1}^{*}$ denotes complex conjugate of $\lambda_{1}$.

We can consider also equilibrium points of near-wall velocity field on the boundary of upper wall, $x= \pm A_{x} / 2$ and $y= \pm A_{y} / 2$, by using approximate velocity fields near this boundary in place of $\left(u_{w}, v_{w}\right)$, as an example of general theory by Surana et al. (2007). These equilibrium points located on the boundary of upper wall can be classified into nodes and saddles, as was explained by Surana et al.

In the present study, we define convection cells in a cavity as those surrounded by boundary surfaces and cavity's walls. Here each boundary surface is defined by the property that the largest distance between the trajectories of two fluid particles starting from locations near this surface on its opposite sides in velocity field $\hat{\boldsymbol{u}}$ remains finite even when the initial distance between these fluid particles decreases to zero. For the velocity field of most unstable mode obtained in the present study, streamlines of an upper-wall flow pattern always originate from an equilibrium point and end at another equilibrium point (That is, no streamline of closed curve is observed in the flow pattern.). Some of these streamlines are important in the examination of geometrical structure of convection cells. That is, streamlines connecting a saddle and an equilibrium point (node, saddle, or spiral) are expected to be upper ends of boundary surfaces defined above, because the rapid divergent motion of fluid particles to opposite directions is expected when they are initially close to these streamlines and pass through a region close to this saddle (under time-reversal if necessary). The validity of this expectation is confirmed by the comparison of upper-wall flow patterns with trajectories of many fluid particles for several aspect ratios.

\subsubsection{Flow patterns for $1 \leq A_{x} \ll A_{y}$}

Although the direction of flow of most unstable mode cannot be determined in the 


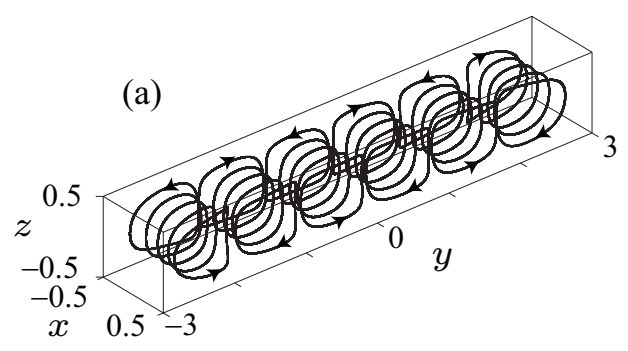

(b)

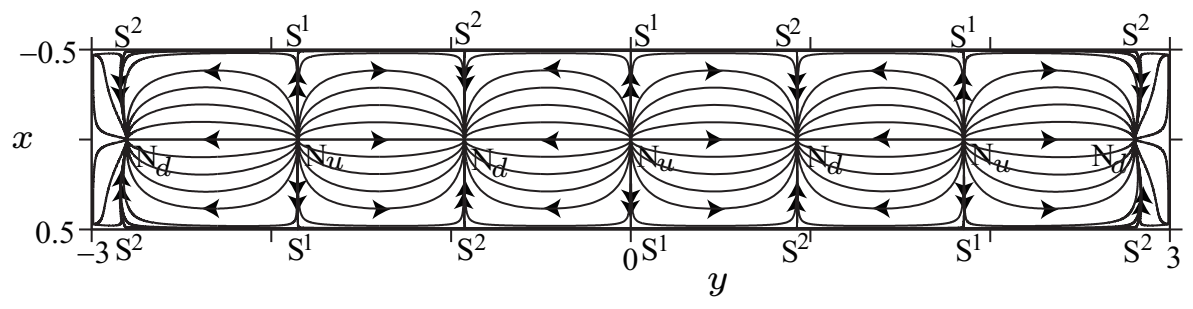

(c)

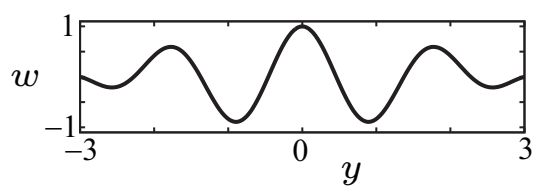

(d)

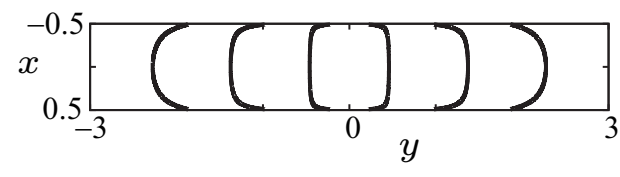

Figure 5. Characteristics of the flow of most unstable mode for $\left(A_{x}, A_{y}\right)=(1,6)$. (a) Trajectories of several fluid particles. (b) Upper-wall flow pattern. $\mathrm{N}_{u}$ and $\mathrm{N}_{d}$ denote upward and downward nodes of near-wall velocity field, whereas $S^{1}$ and $S^{2}$ denote saddles on the boundary of upper wall. (c) Dependence of $w$ on $y$ at $x=z=0$. (d) Axis lines of circulation of convection cells.

linear stability analysis, in the following part of present paper, flow patterns are usually explained on the basis of the velocity field of a selected direction so that the explanation is simpler.

If condition $1 \leq A_{x} \ll A_{y}$ is satisfied, the flow pattern of most unstable mode in the region not close to side walls is a linear arrangement of several convection cells whose axes of circulation are horizontal and parallel to shorter side walls $y= \pm A_{y} / 2$, consistently with the results obtained in preceding studies. An example of such flow pattern is shown in Fig. 5(a) for $\left(A_{x}, A_{y}\right)=(1,6)$ by closed trajectories of several fluid particles whose initial locations are not close to side walls. Six convection cells are observed for this $\left(A_{x}, A_{y}\right)$.

Figure 5(b) shows an upper-wall flow pattern for $\left(A_{x}, A_{y}\right)=(1,6)$. There are four downward nodes denoted by $\mathrm{N}_{d}$ and three upward nodes denoted by $\mathrm{N}_{u}$ on the line $x=0$, and also seven pairs of saddles denoted by $\mathrm{S}^{1}$ and $\mathrm{S}^{2}$ on the boundaries $x= \pm 0.5$. Most streamlines of this flow pattern originate from one of $\mathrm{N}_{u}$ 's and end at a neighboring $\mathrm{N}_{d}$. These streamlines are approximately parallel to the boundaries $x= \pm 0.5$ as long as they are far from these nodes, but bend inwardly when they approach these nodes. 
Moreover, we also observe four pairs of streamlines originating from $\mathrm{S}^{2}$ and ending at $\mathrm{N}_{d}$, and three pairs of streamlines from $\mathrm{N}_{u}$ to $\mathrm{S}^{1}$, expressed by duplicated arrowheads in Fig. 5(b). These streamlines are almost straight and approximately perpendicular to the boundaries $x= \pm 0.5$. They are upper ends of boundary surfaces of six convection cells shown in Fig. 5(a).

The ascending or descending flows near the boundaries of convection cells are stronger in the central region of cavity than in the region near shorter side walls, as expected from the stronger effect of rigid side walls in the latter region. This property is illustrated in Fig. 5(c) where dependence of $w$ on $y$ for $x=z=0$ is shown. Here $w$ is normalized so that it is 1 at $y=0$. We observe a rapid decrease in the strength of ascending or descending flows as the walls $y= \pm 3$ are approached, although it is difficult to recognize this property from the trajectories in Fig. 5(a). This property is confirmed by the decrease in $\left|D_{w}\right|$ at the nodes of upper-wall flow pattern as their $|y|$ values increase. That is, the values of $D_{w}$ at the nodes of $y=0,0.925,1.852$ and 2.805 in Fig. 5(b) are 1, $-0.887,0.571$ and -0.095 , respectively, when they are normalized so that $D_{w}=1$ at the node of $y=0$.

The $y$ coordinates of local maxima or minima of $w$ in Fig. $5(\mathrm{c})$ are $y_{m}^{(0)}=0, y_{m}^{(1)}=$ $0.895, y_{m}^{(2)}=1.772$, and $y_{m}^{(3)}=2.570$. Because these values are a little smaller than the $y$ coordinates of nodes of upper-wall flow pattern shown above except for zero, it is suggested that the boundary surfaces of convection cells are vertically a little concave toward the direction of increasing $|y|$. The width-to-height ratios of convection cells based on $y_{m}^{(j)}(j=0, \ldots, 3)$ are $0.895,0.877$ and 0.798 , for inside, middle and outside cells, respectively. These ratios are a little smaller than 1 , and the ratio of outside cells is especially small. Moreover, the ratio 0.895 for the inside cells is close to the widthto-height ratio 0.929 of the convection cells of most unstable mode at critical Rayleigh number for an infinite channel of square cross section obtained by Daniels and Ong (1990).

We define the axis of circulation in each convection cell by a line where $|\hat{\boldsymbol{u}}|$ is approximately zero. The axis line is computed by extending a line toward the direction of smallest $|\hat{\boldsymbol{u}}|$ from the point of $|\hat{\boldsymbol{u}}|=0$ on the line $x=z=0$. The axis lines for the flow of Fig. 5(a) obtained by this method are shown in Fig. 5(d) (The values of $|\hat{\boldsymbol{u}}|$ on these lines are found to be almost zero.). They are always on the horizontal central plane $z=0$, and are almost straight for the inside cells unless they are close to the side walls $x= \pm 0.5$, but are strongly curved for the outside cells.

If $A_{y}$ increases from $A_{x}$ for fixed $A_{x}$ larger than 1, the number of convection cells increases by one as $A_{y}$ crosses the boundaries of most unstable mode shown in Fig. 3. For example, for $A_{x}=2$, the flow patterns of most unstable mode are composed of 3 , 4 and 5 convection cells if conditions $2.801 \leq A_{y} \leq 3.873,3.873 \leq A_{y} \leq 4.947$ and $4.947 \leq A_{y} \leq 6.019$ are satisfied, respectively. Although the widths of these cells based on the local maxima and minima of $w$ on the line $x=z=0$ increase with $A_{y}$ within each $A_{y}$ region of fixed number of cells, they are restricted to the range between 0.80 and 1.05. Therefore, the width-to-height ratio of convection cells is a little smaller than 
1 for most $A_{y}$. Moreover, the width of outermost convection cells is usually smaller than that of other cells by about $10-20 \%$, which causes relatively small width-to-height ratio of the outermost cells.

In the experiments by Stork and Müller (1972), the flow pattern that appears first as $R$ is increased slowly was examined through top-view images of fluid motion in a cavity visualized by aluminum flakes. Although the correspondence between these images and actual flow patterns is not clear, it seems reasonable to assume that the direction of fluid velocity is roughly horizontal in white parts of the images, and is roughly vertical in their black parts. If this assumption is adopted, the flow patterns of most unstable mode in the region not close to side walls obtained in the present study for $1 \leq A_{x} \ll A_{y}$ are consistent with their experimental results. That is, several white thick lines parallel to shorter side walls in the experimental images for such $\left(A_{x}, A_{y}\right)$ suggest a linear arrangement of convection cells whose axes of circulation are parallel to shorter side walls. However, more detailed comparison of the characteristics of flow patterns obtained in the present study with experimental results is difficult because the velocity of convective motion was not measured by Stork and Müller (1972). Although there is a tendency in the images by Stork and Müller (1972) that white thick lines near shorter side walls are convex outwardly, its relation to the curved axis lines of convection cells near shorter side walls shown in Fig. 5(d) is not clear.

Stork and Müller (1972) obtained also the result that the vertical cross-section of convection cells at the onset of convection is nearly squared unless either of two aspect ratios is small. Although this result is consistent with our results for $1 \leq A_{x} \ll A_{y}$, more detailed comparison is difficult because of the lack in the accurate data of widthto-height ratio of each convection cell in experiments. Stork and Müller (1972) examined also the boundaries of $\left(A_{x}, A_{y}\right)$ at which the number of convection cells at the onset of convection changes. For $A_{x}=2$, they observed that this number increases from 2 to 3,4 and 5 at $A_{y}$ 's within the ranges of $2.5-2.7,3.3-3.9$ and $4.9-5.1$, respectively. Corresponding $A_{y}$ values, 2.801,3.873 and 4.947, obtained in the present study are within these ranges or slightly above them, which suggests rough consistency between experimental and numerical results.

Davis (1967) numerically obtained the boundary lines of $\left(A_{x}, A_{y}\right)$ on which the number of convection cells of most unstable mode changes, under the assumption of finite roll. For $A_{x}=2$, the values of $A_{y}$ at which this number increases from 2 to 3, 4 and 5 are found to be about 2.8, 3.6 and 4.6, respectively, from Fig. 13 of his paper. These values are slightly smaller than our values mentioned above except for the first one. The main reason for this slight discrepancy seems the use of assumption of finite roll in Davis' study. In order to examine the validity of this assumption for $1 \leq A_{x} \ll A_{y}$, the following index $\Gamma$ is introduced:

$$
\Gamma=\frac{\max _{x, y, z}|u(x, y, z)|}{\max _{x, y, z}|\hat{\boldsymbol{u}}(x, y, z)|} .
$$

If $A_{x}$ is fixed to 1 , the values of $\Gamma$ are $0.129,0.120,0.119$ and 0.118 for $A_{y}=2,4,6$ and 
9, respectively. Therefore, since it is suggested that $\Gamma$ asymptotes to a value close to 0.118 as $A_{y}$ tends to infinity, the validity of assumption of finite roll is limited even if $A_{y}$ is much larger than $A_{x}$.
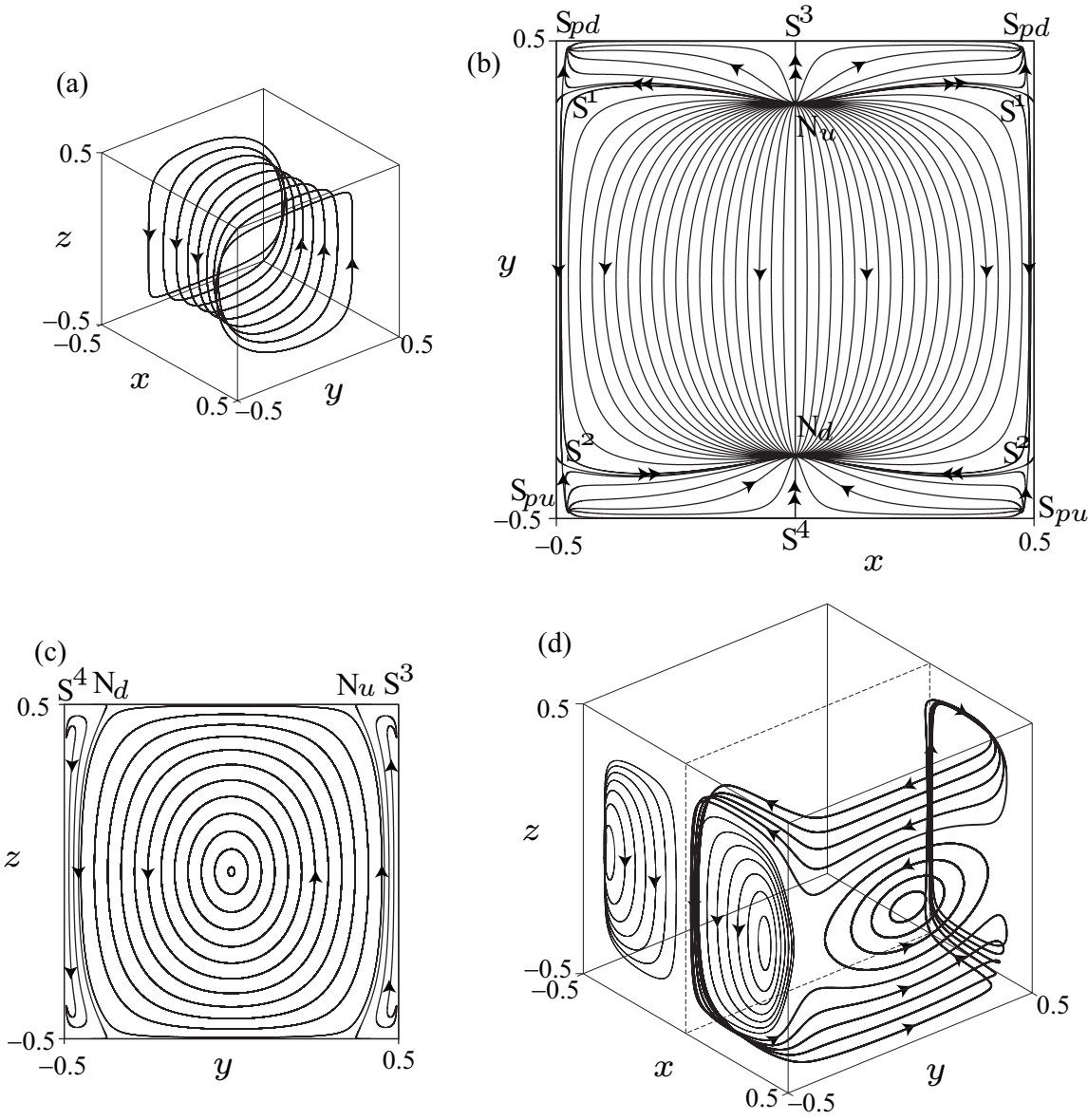

Figure 6. Characteristics of the flow of most unstable $\hat{\theta}(e, o, e)$ mode for $A=1$. (a) Trajectories of fluid particles starting from $y=0$ and $z=0.4$ with $x=$ $-0.4,-0.3, \ldots, 0.4$. (b) Upper-wall flow pattern. $\mathrm{N}_{u}$ and $\mathrm{N}_{d}$ denote upward and downward nodes, respectively, $\mathrm{S}^{j}(j=1, \ldots, 4)$ denote saddles, and $\mathrm{S}_{p u}$ and $\mathrm{S}_{p d}$ denote upward and downward spirals, respectively. (c) Trajectories of fluid particles on the plane $x=0$. (d) Trajectories of fluid particles starting from $x=0.49$ and $y=0$ with $z=0.05,0.10, \ldots, 0.45$ (thick lines), and those starting from $y=-0.497$ and $z=0$ with $x= \pm 0.05, \pm 0.10, \ldots, \pm 0.35$ (thin lines).

5.2.2 Flow patterns for $1 \leq A_{x}=A_{y} \leq 6$

We next examine the flow pattern of most unstable mode in a cavity of square base in which $A_{x}=A_{y}=A \geq 1$. For a cubic cavity of $A=1$, the flow pattern in the central region of cavity is a roll whose axis of circulation is horizontal and parallel to a side wall, as shown in Fig. 6(a) by trajectories of several fluid particles. This axis is parallel to the walls $y= \pm 0.5$ for $\hat{\theta}(e, o, e)$ mode (as shown in Fig. 6(a)), or to the walls $x= \pm 0.5$ for 
$\hat{\theta}(o, e, e)$ mode. The flow pattern of the latter mode is obtained by the rotation of that of the former mode by $\pi / 2$ around the central vertical line of cavity. Mizushima and Matsuda (1997) showed a schematic flow pattern and projections of velocity fields on a few cross-sections for the most unstable mode in a cubic cavity. Although their flow pattern in the central region of cavity is consistent with Fig. 6(a), the detailed structure of flow pattern near side walls was not shown by them.

The upper-wall flow pattern of the most unstable $\hat{\theta}(e, o, e)$ mode for $A=1$ is shown in Fig. 6(b). Streamlines in the central part of this figure originate from an upward node $\mathrm{N}_{u}$ and end at a downward node $\mathrm{N}_{d}$. These streamlines correspond to the motion of a fluid at the upper end of roll pattern shown in Fig. 6(a). We also observe four saddles $\mathrm{S}^{1}$ and $\mathrm{S}^{2}$ near the boundaries $x= \pm 0.5$, and two saddles $\mathrm{S}^{3}$ and $\mathrm{S}^{4}$ on the boundaries $y= \pm 0.5$, There are streamlines originating from $\mathrm{S}^{2}$ and ending at $\mathrm{N}_{d}$, and those originating from $\mathrm{N}_{u}$ and ending at $\mathrm{S}^{1}$, expressed by duplicated arrowheads in Fig. 6(b). These streamlines are upper ends of boundary surfaces of the main convection cell within which circulating motions of fluid particles around the axis $y=z=0$, such as the trajectories shown in Fig. 6(a), are observed. Figure 6(c) shows the trajectories of fluid particles on the symmetry plane $x=0$. The cross-section of boundary surfaces of the main convection cell, the curves intersecting with the upper boundary $z=0.5$ at $\mathrm{N}_{d}$ and $\mathrm{N}_{u}$, is found in this figure.

The motion of fluid particles that are within the main convection cell but are close to its boundary surfaces or side walls $x= \pm 0.5$ can be much different from the roll pattern shown in Fig. 6(a). That is, as expressed by thick lines in Fig. 6(d), fluid particles starting from the locations near the side wall $x=0.5$ move along closed orbits that are always close to this side wall if $|z|$ of their initial locations is small. However, they move along closed orbits that pass through also the regions close to other side walls $y= \pm 0.5$ if this $|z|$ is large (trajectories of fluid particles starting from the locations near the opposite side wall $x=-0.5$ are just the reflection of the above trajectories with respect to the plane $x=0$ because of the symmetry of $\hat{\boldsymbol{u}}$ ). In order to clarify the difference between these two motions, we define a quasi two-dimensional trajectory by the property that the difference between the maximum and minimum values of $x$ on this trajectory is smaller than a small threshold value of $0.02 \mathrm{~A}$. Trajectories of the former motion are quasi two-dimensional, but those of the latter motion are not. Here we also define the region of quasi two-dimensional motion as the region occupied by all the quasi two-dimensional trajectories within the main convection cell. From Figs. 6(c) and (d), it is suggested that this region extends from the symmetry plane $x=0$ to the side walls $x= \pm 0.5$. Furthermore, in the wall flow pattern on the side wall $x=0.5$, we observe closed streamlines around the point $y=z=0$ that are similar to four closed orbits around the line $y=z=0$ in Fig. 6(d). The area of region occupied by such streamlines is about $20 \%$ of the total area of this side wall. On the other hand, the cross-section of region of quasi two-dimensional motion on the plane $x=0$ occupies about $85 \%$ of the area of this plane, as found from Fig. 6(c). Therefore, although the region of quasi two-dimensional motion surely extends to the side walls $x= \pm 0.5$, 
the area of its cross-section near these side walls is considerably smaller than the area on the symmetry plane $x=0$. Trajectories of fluid particles that are within the main convection cell but pass through the region close to its boundary surfaces are not quasi two-dimensional, as illustrated in Fig. 6(d). This behavior is consistent with streamlines near the streamlines from $\mathrm{N}_{u}$ to $\mathrm{S}^{1}$ or from $\mathrm{S}^{2}$ to $\mathrm{N}_{d}$ in the upper-wall flow pattern of Fig. 6(b).

In Fig. 6(b), we observe two small regions near the boundaries $y= \pm 0.5$ where most streamlines originate from $\mathrm{N}_{u}$ and end at either of two downward spirals denoted by $\mathrm{S}_{p d}$ near upper corners, or originate from either of two upward spirals denoted by $\mathrm{S}_{p u}$ near lower corners and end at $\mathrm{N}_{d}$. These regions are upper ends of three-dimensional thin regions, which we call wall regions, delimited by the boundary surfaces of main convection cell and side walls of cavity. Typical trajectories of fluid particles in the wall regions are closed orbits that are always close to the side walls $y= \pm 0.5$. Their examples are shown in Fig. 6(d) with thin lines. As found from these trajectories, each of the wall regions is divided into two convection cells by the plane $x=0$. In Fig. 6(b), we observe saddles denoted by $\mathrm{S}^{3}$ and $\mathrm{S}^{4}$ at $(x, y)=(0, \pm 0.5)$, and also streamlines connecting $\mathrm{S}^{3}$ and $\mathrm{N}_{u}$ and connecting $\mathrm{S}^{4}$ and $\mathrm{N}_{d}$. These streamlines are upper ends of boundary surface $x=0$ of the two pairs of convection cells composing the wall regions.

Trajectories that are within the main convection cell but are not quasi twodimensional and trajectories within the wall regions are far from those expected under the assumption of finite roll. However, the value of $\Gamma$ for the velocity field considered is 0.094 , which is relatively small and slightly smaller than the $\Gamma$ for $A_{x}=1, A_{y} \gg 1$. This small $\Gamma$ is explained by the small value of $|\hat{\boldsymbol{u}}|$ near side walls, where $|u|$ may as large as $|v|$ and $|w|$, compared with $|\hat{\boldsymbol{u}}|$ in the central region of cavity.

Flow patterns of most unstable mode similar to Fig. 6 are observed for $A$ satisfying $1 \leq A \leq 1.312$, the upper bound of which corresponds to $\mathrm{M}_{1}$ in Fig. 3. As $A$ increases from 1 within this range, nodes $\mathrm{N}_{d}$ and $\mathrm{N}_{u}$ and saddles $\mathrm{S}^{1}$ and $\mathrm{S}^{2}$ in upper-wall flow patterns gradually move away from the boundaries $y= \pm A / 2$. For example, the $y$ coordinate of $\mathrm{N}_{u}$ is $74 \%$ of $A / 2$ for $A=1$, whereas it is $67 \%$ for $A=1.3$. Therefore, the volume ratio of main convection cell to the cavity decreases with increasing $A$. Moreover, the volume ratio of the region of quasi two-dimensional motion to the cavity decreases more rapidly as $A$ increases. For example, closed streamlines around the point $y=z=0$ are not observed in the wall flow pattern on $x=A / 2$ for $A=1.3$, which means that the region of quasi two-dimensional motion does not extend to the side walls $x= \pm A / 2$. The value of $\Gamma$ increases with $A$ and reaches 0.161 for $A=1.3$. Therefore, the assumption of finite roll is expected to be less valid for larger $A$.

For $1.312 \leq A \leq 2.968, \hat{\theta}(e, e, e)$ mode is the most unstable. Typical trajectories of fluid particles by $\hat{\boldsymbol{u}}$ of this mode are shown in Fig. 7(a) for $A=2$. These trajectories reveal radial circulation with a strong ascending flow in the central part of cavity. The motion of fluid particles starting from one of the four vertical planes, $x=0, y=0$ and $y= \pm x$, is restricted to this plane.

The upper-wall flow pattern of this $\hat{\boldsymbol{u}}$ is shown in Fig. 7(b). At the central point 
(a)

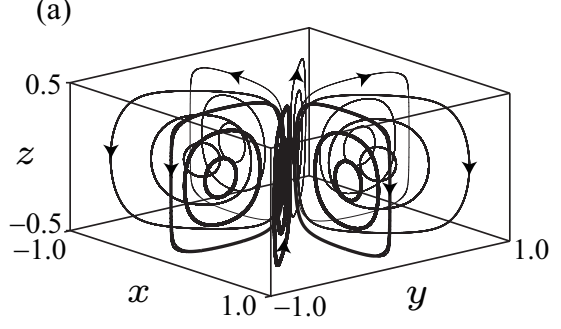

(b)

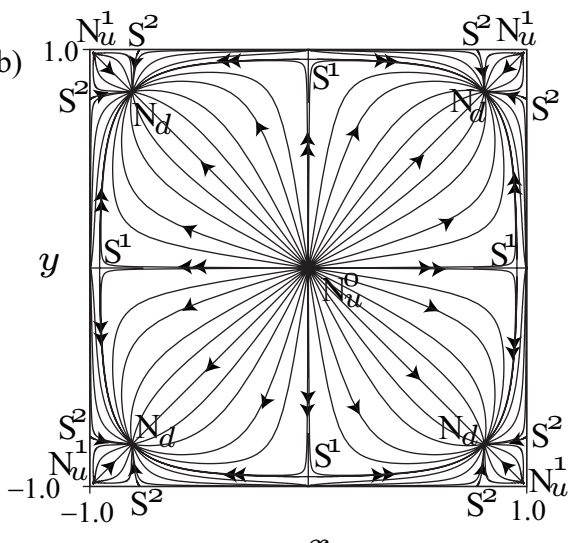

$x$

Figure 7. Characteristics of the flow of most unstable mode for $A=2$. (a) Trajectories of fluid particles on four vertical planes $x=0, y=0$ and $y= \pm x$. Trajectories closer to view point are drawn with thicker lines. (b) Upper-wall flow pattern. $\mathrm{N}_{u}^{j}(j=0,1)$ and $\mathrm{N}_{d}$ denote upward and downward nodes of near-wall velocity field, respectively, whereas $\mathrm{S}^{1}$ and $\mathrm{S}^{2}$ denote saddles.

$x=y=0$, we observe an upward node $\mathrm{N}_{u}^{0}$ corresponding to the ascending flow in the central part of cavity. There are also four saddles $S^{1}$ that are close to the boundary of upper wall and on the line $x=0$ or $y=0$, four downward nodes $\mathrm{N}_{d}$ and four upward nodes $\mathrm{N}_{u}^{1}$ on diagonal lines $y= \pm x$, and eight saddles $\mathrm{S}^{2}$ on the boundary. Most streamlines originate from $\mathrm{N}_{u}^{0}$ and end at one of $\mathrm{N}_{d}$ 's if they are not close to the boundaries $x= \pm 1$ and $y= \pm 1$. The region within a cavity is divided into four central convection cells and small convection cells near side walls. The upper ends of boundary surfaces of central convection cells are composed of streamlines from $\mathrm{N}_{u}^{0}$ to $\mathrm{S}^{1}$ and streamlines from $\mathrm{S}^{1}$ to $\mathrm{N}_{d}$, expressed by duplicated arrowheads in Fig. 7(b). From the streamlines near the boundaries in Fig. 7(b), it is suggested that the motion of fluid particles near side walls is generally not similar to that in the central part of cavity, like the case of $A=1$. An example of such motion is the circulation near vertical corner lines.

Stork and Müller (1972) showed a figure of top-view image of fluid motion for $A=2$ obtained in their experiments. A central black region surrounded by an annular white region shown in this figure is consistent with the strong vertical flow at the center of cavity shown in Fig. 7(a).

For $2.968 \leq A \leq 3.524, \hat{\theta}(e, o, e)$ (and $\hat{\theta}(o, e, e))$ mode is the most unstable, as in the case of $1 \leq A \leq 1.312$. Typical trajectories by $\hat{\boldsymbol{u}}$ of $\hat{\theta}(e, o, e)$ mode are shown in Fig. 8(a) for $A=3$. Fluid particles starting from the vertical plane $x=0$ are always on this plane. Upper-wall flow pattern of this $\hat{\boldsymbol{u}}$ is shown in Fig. 8(b). In the central region of this flow pattern, we observe upward and downward nodes $\mathrm{N}_{u}^{1}$ and $\mathrm{N}_{d}^{1}$ on the line $x=0$. There are also two pairs of downward nodes denoted by $\mathrm{N}_{d}^{2}$ and $\mathrm{N}_{d}^{3}$, and two pairs of upward nodes denoted by $\mathrm{N}_{u}^{2}$ and $\mathrm{N}_{u}^{3}$, near the corners. Moreover, near the boundaries $x= \pm 1.5$ and $y= \pm 1.5$, we find several saddles $S^{j}(j=1, \ldots, 8)$. Most 


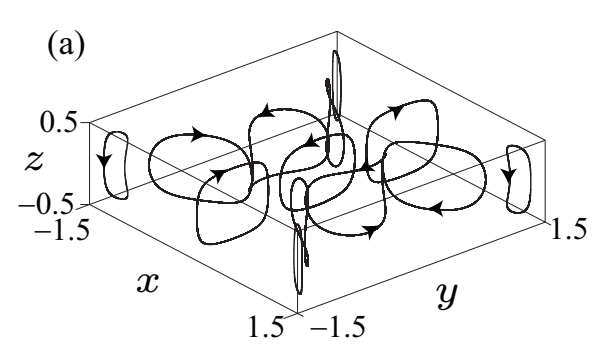

(b)

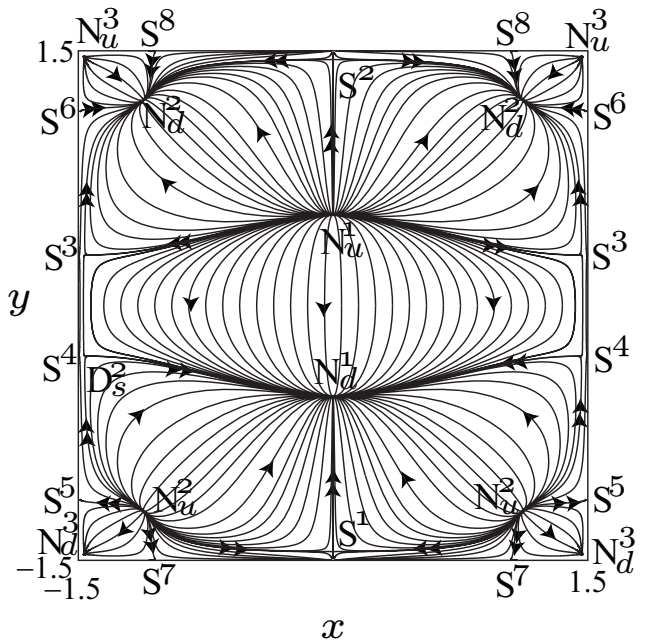

Figure 8. Characteristics of the flow of most unstable $\hat{\theta}(e, o, e)$ mode for $A=3$. (a) Trajectories of several fluid particles. (b) Upper-wall flow pattern. $\mathrm{N}_{u}^{j}(j=1,2,3)$ and $\mathrm{N}_{d}^{j}(j=1,2,3)$ denote upward and downward nodes of near-wall velocity field, respectively, whereas $\mathrm{S}^{j}(j=1, \ldots, 8)$ denote saddles.

streamlines originate from $\mathrm{N}_{u}^{1}$ or $\mathrm{N}_{u}^{2}$ and end at $\mathrm{N}_{d}^{1}$ or $\mathrm{N}_{d}^{2}$ except for streamlines near the boundary. The region within a cavity is divided into a central convection cell, four convection cells adjacent to this cell, and small convection cells close to side walls. The upper ends of boundary surfaces of central convection cell are streamlines from $\mathrm{N}_{u}^{1}$ to $\mathrm{S}^{3}$ and from $\mathrm{S}^{4}$ to $\mathrm{N}_{d}^{1}$, expressed by duplicated arrowheads in Fig. 8(b). The motion of fluid particles within this convection cell is roughly the circulation around the axis $y=z=0$, similarly to the motion within the main convection cell for $A=1$. The upper ends of boundary surfaces of four adjacent convection cells are composed of the above streamlines and the streamlines connecting saddles $\mathrm{S}^{j}(j=1, \ldots, 4)$ and their neighboring nodes $\mathrm{N}_{d}^{2}$ and $\mathrm{N}_{u}^{2}$ expressed again by duplicated arrowheads. Within these adjacent convection cells, it is roughly said that fluid particles move around an axis that is on the plane $z=0$ and is not parallel to any side walls, like the lines connecting $\mathrm{S}^{2}$ $\left(\mathrm{S}^{1}\right)$ and $\mathrm{S}^{3}\left(\mathrm{~S}^{4}\right)$. The motion of fluid particles near the side walls $y= \pm 1.5$ is different from that in the central and adjacent convection cells. For example, we observe small circulating motion in convection cells close to vertical corner lines of cavity, illustrated in Fig. 8(a).

Of several equilibrium points shown in Fig. 8(b), two nodes $\mathrm{N}_{u}^{1}$ and $\mathrm{N}_{d}^{1}$ located near the center are important because $|\hat{\boldsymbol{u}}|$ is expected to be relatively large in the central region of cavity. $\left|D_{w}\right|$ at these nodes is at least 9 times as large as $\left|D_{w}\right|$ at other equilibrium points. Therefore, strong ascending or descending flows in the vertical cylindrical regions around $\mathrm{N}_{u}^{1}$ and $\mathrm{N}_{d}^{1}$ are expected. As suggested from Fig. 8(b), the deviation of trajectories of fluid particles from those expected under the assumption of finite roll is not small even in the central region of cavity where $|\hat{\boldsymbol{u}}|$ is relatively large. This results in the large value of $\Gamma=0.341$ for the $\hat{\boldsymbol{u}}$ of Fig. 8, which is much larger 
than the $\Gamma$ for $A=1$. Therefore, we can no longer expect the validity of finite-roll assumption for $A=3$.

(a)

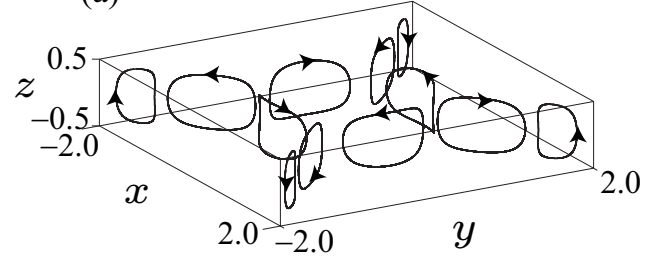

(b)

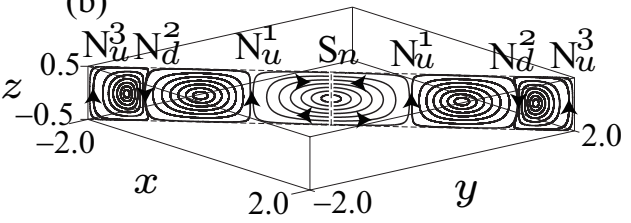

(c)

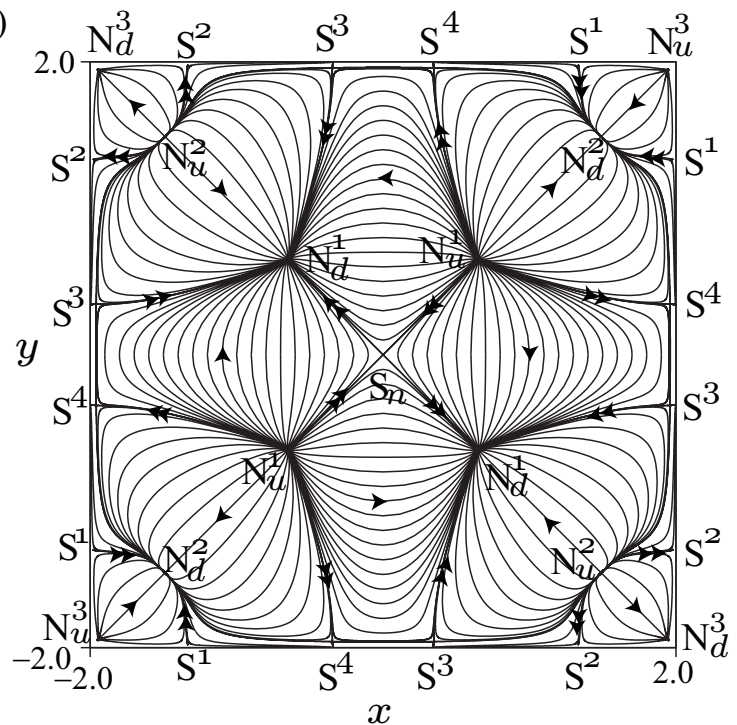

(d)

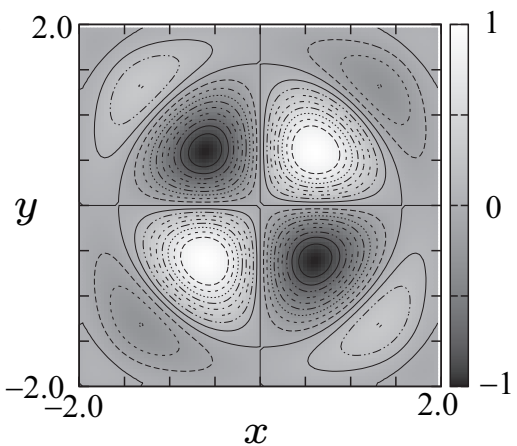

Figure 9. Characteristics of the flow of most unstable mode for $A=4$. (a) Trajectories of several fluid particles. (b) Trajectories of fluid particles on the vertical diagonal plane $y=x$. (c) Upper-wall flow pattern. $\mathrm{N}_{u}^{j}$ and $\mathrm{N}_{d}^{j}(j=1,2,3)$ denote upward and downward nodes of near-wall velocity field, respectively, $\mathrm{S}^{j}(j=1, \ldots, 4)$ denote upward or downward saddles, and $\mathrm{S}_{n}$ denotes a neutral saddle. (d) Distribution of $w$ on the horizontal plane $z=0$. Its value is normalized so that largest $|w|$ is 1 .

For $3.524 \leq A \leq 4.418$, the most unstable mode is $\hat{\theta}(o, o, e)$ mode, which is not the most unstable if the difference between $A_{x}$ and $A_{y}$ is large. Typical trajectories of fluid particles by $\hat{\boldsymbol{u}}$ of this mode are shown in Fig. 9(a) for $A=4$. The motion of fluid particles starting from one of the diagonal vertical planes $y= \pm x$ is restricted to this plane. In Fig. 9(b) of the motion of fluid particles on the plane $y=x$, we observe trajectories starting from points close to the central vertical line $x=y=0$ where $\hat{\boldsymbol{u}}$ is zero, and approaching different points on this line, as well as circulating trajectories in the regions closer to vertical corner lines.

The upper-wall flow pattern of this $\hat{\boldsymbol{u}}$ is shown in Fig. 9(c). A neutral saddle $\mathrm{S}_{n}$ at the center is surrounded by two pairs of downward nodes $\mathrm{N}_{d}^{1}$ and upward nodes $\mathrm{N}_{u}^{1}$ on 
diagonal lines. On these lines, there are also four pairs of downward nodes $\mathrm{N}_{d}^{2}$ and $\mathrm{N}_{d}^{3}$ and upward nodes $\mathrm{N}_{u}^{2}$ and $\mathrm{N}_{u}^{3}$ close to the corners. Moreover, we observe several saddles $\mathrm{S}^{j}(j=1, \ldots, 4)$ near or on the boundaries $x= \pm 2$ and $y= \pm 2$. Most streamlines originate from $\mathrm{N}_{u}^{1}, \mathrm{~N}_{u}^{2}$ or $\mathrm{N}_{u}^{3}$, and end at $\mathrm{N}_{d}^{1}, \mathrm{~N}_{d}^{2}$ or $\mathrm{N}_{d}^{3}$. The region within a cavity is occupied by 12 convection cells of three kinds. The upper ends of boundary surfaces of four central convection cells are composed of streamlines from $\mathrm{S}^{3}$ to $\mathrm{N}_{d}^{1}$ and from $\mathrm{N}_{u}^{1}$ to $\mathrm{S}^{4}$, and streamlines connecting $\mathrm{S}_{n}$ and $\mathrm{N}_{d}^{1}$ or $\mathrm{N}_{u}^{1}$. These convection cells almost extend to side walls. The motion of fluid particles within these cells is roughly the circulation around the axis $x=z=0$ or $y=z=0$. There are also four convection cells called corner convection cells near vertical corner lines. The upper ends of their boundary surfaces are streamlines connecting $\mathrm{N}_{d}^{2}\left(\mathrm{~N}_{u}^{2}\right)$ and $\mathrm{S}^{1}\left(\mathrm{~S}^{2}\right)$. Typical trajectories within these convection cells are closed orbits near vertical corner lines shown in Fig. 9(a). Remaining four convection cells called middle convection cells are located between central and corner convection cells. Fluid particles within middle convection cells move around the axis that is on the plane $z=0$ and is approximately specified by $y= \pm x \pm 2.3$. All the streamlines that are upper ends of boundary surfaces of 12 convection cells are expressed by duplicated arrowheads in Fig. 9(b). In Fig. 9(a), trajectories of 12 representative fluid particles within each of these convection cells are shown.

The value of $\left|D_{w}\right|$ at nodes $\mathrm{N}_{u}^{1}$ and $\mathrm{N}_{d}^{1}$ is more than 5 times as large as $\left|D_{w}\right|$ at other equilibrium points of near-wall velocity field. Therefore, strong ascending or descending flows are expected in the vertical cylindrical regions around these nodes. This is confirmed by the distribution of $w$ on the central horizontal plane $z=0$ shown in Fig. 9(d). We observe strong ascending or descending flows near the locations of $\mathrm{N}_{u}^{1}$ and $\mathrm{N}_{d}^{1}$ in Fig. 9(c). Stork and Müller (1972) showed a figure of top-view image of fluid motion for $A=4$. Four black regions on diagonal lines shown in this figure are consistent with the regions of large $|w|$ in Fig. 9(d).

For $4.418 \leq A \leq 5.023$, the most unstable mode is again $\hat{\theta}(e, o, e)($ and $\hat{\theta}(o, e, e))$ mode. From trajectories of several fluid particles by $\hat{\boldsymbol{u}}$ of $\hat{\theta}(e, o, e)$ mode and an upperwall flow pattern of this $\hat{\boldsymbol{u}}$ for $A=5$ shown in Figs. 10(a) and (b), we find that the flow pattern for $A=5$ is more complicated and is composed of more convection cells than the flow pattern for $A=3$ of the same symmetry. In the central region of Fig. 10(b), there are upward nodes $\mathrm{N}_{u}^{1}$ and $\mathrm{N}_{u}^{2}$, downward nodes $\mathrm{N}_{d}^{1}$ and $\mathrm{N}_{d}^{2}$, and a pair of neutral saddles $\mathrm{S}_{n}$, in addition to saddles $\mathrm{S}^{1}$ and $\mathrm{S}^{2}$. There are also several nodes denoted by $\mathrm{N}_{d}^{j}$ and $\mathrm{N}_{u}^{j}(j=3,4,5)$ and several saddles denoted by $\mathrm{S}^{j}(j=3, \ldots, 10)$ near the boundary. The region within a cavity is divided into 19 convection cells. The upper ends of boundary surfaces of these cells are composed of several streamlines connecting saddles and equilibrium points denoted by duplicated arrowheads in Fig. 10(b). As found from trajectories of 19 representative fluid particles within each of these convection cells shown in Fig. 10(a), fluid particles move around an axis located on the horizontal plane $z=0$ in each convection cell. Although some of the axes are straight, the remaining axes are a little convex outward. It is suggested from Fig. 10(b) that a central convection cell and four adjacent convection cells do not extend to side walls, unlike the case of $A=3$. 

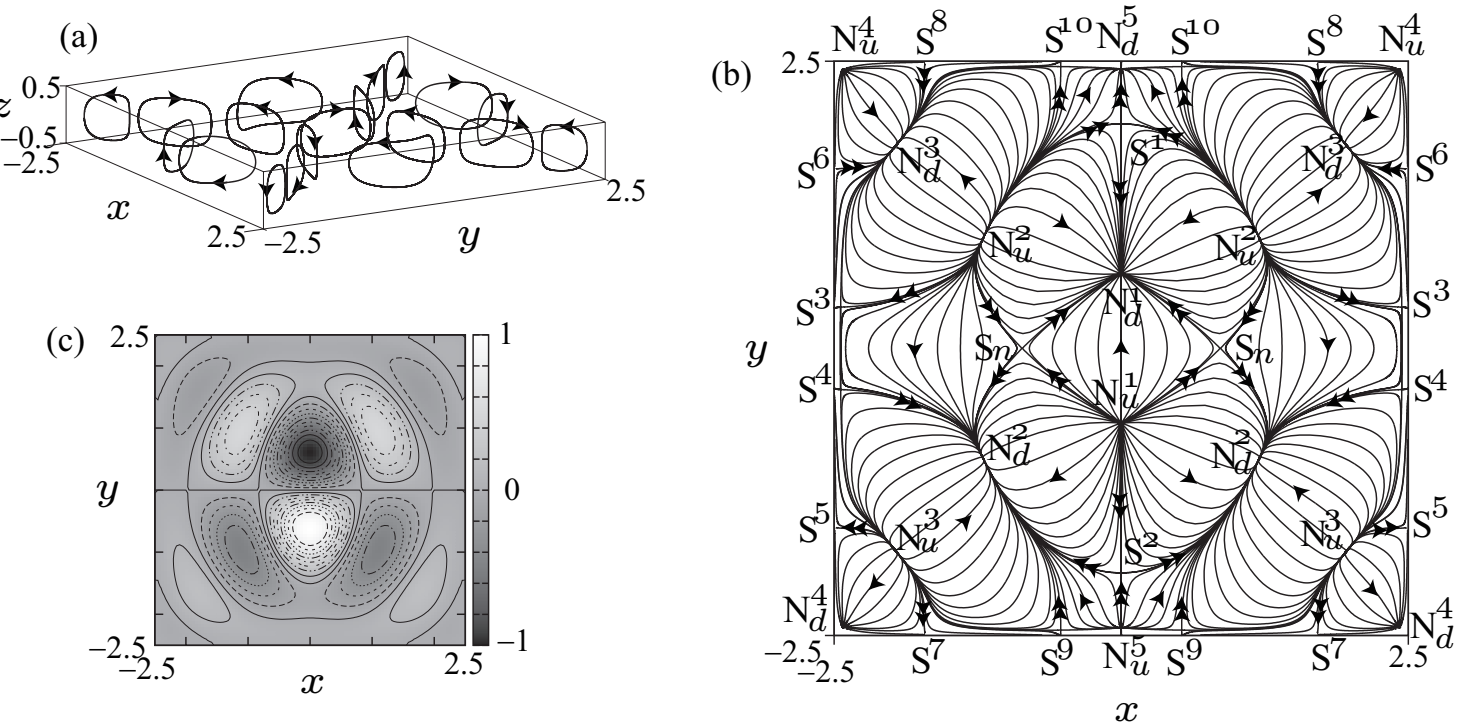

Figure 10. Characteristics of the flow of most unstable $\hat{\theta}(e, o, e)$ mode for $A=5$. (a) Trajectories of several fluid particles. (b) Upper-wall flow pattern. $\mathrm{N}_{u}^{j}$ and $\mathrm{N}_{d}^{j}(j=1, \ldots, 5)$ denote upward and downward nodes of near-wall velocity field, respectively, $\mathrm{S}^{j}(j=1, \ldots, 10)$ denote upward or downward saddles, and $\mathrm{S}_{n}$ denotes a neutral saddle. (c) Distribution of $w$ on the horizontal plane $z=0$. Its value is normalized so that largest $|w|$ is 1 .

Of the $\left|D_{w}\right|$ 's at equilibrium points shown in Fig. 10(b), $\left|D_{w}\right|$ at the nodes closest to the center, $\mathrm{N}_{d}^{1}$ and $\mathrm{N}_{u}^{1}$, is the largest, and is almost twice the $\left|D_{w}\right|$ at neighboring nodes $\mathrm{N}_{d}^{2}$ and $\mathrm{N}_{u}^{2}$. The values of $\left|D_{w}\right|$ at other equilibrium points are less than one third of $\left|D_{w}\right|$ at $\mathrm{N}_{d}^{2}$ and $\mathrm{N}_{u}^{2}$. Therefore, the strongest and the second strongest vertical flows are expected in the vertical cylindrical regions around $\mathrm{N}_{d}^{1}$ and $\mathrm{N}_{u}^{1}$, and around $\mathrm{N}_{d}^{2}$ and $\mathrm{N}_{u}^{2}$, respectively. These flows are confirmed by the distribution of $w$ on the horizontal plane $z=0$ shown in Fig. 10(c). In the experiments by Stork and Müller (1972), it was reported that symmetrical arrangements of convection cells or rolls parallel to the diagonal of the rectangular frame were observed for $\left(A_{x}, A_{y}\right)=(5,5.1)$. However, since the figure of this flow pattern was not shown in their paper (probably by a mistake in its publication), the detailed comparison with our results cannot be made.

For $5.023 \leq A \leq 6$, the most unstable mode is $\hat{\theta}(o, o, e)$ mode, like the case of $3.524 \leq A \leq 4.418$. A complicated flow pattern composed of many convection cells for $A=6$ is recognized in Fig. 11(a) showing trajectories of several fluid particles and in Fig. 11(b) showing an upper-wall flow pattern. Twenty convection cells, whose boundary surfaces are delimited at their upper end by streamlines connecting saddles and equilibrium points expressed by duplicated arrowheads in Fig. 11(b), are observed within the region of cavity. In each convection cell, fluid particles move around a straight or curved axis located on the plane $z=0$, as illustrated in Fig. 11(a). Similarly to the case of $A=4$, there is a neutral saddle $S_{n}^{0}$ at the center of Fig. 11(b), and the motion of 
(a)

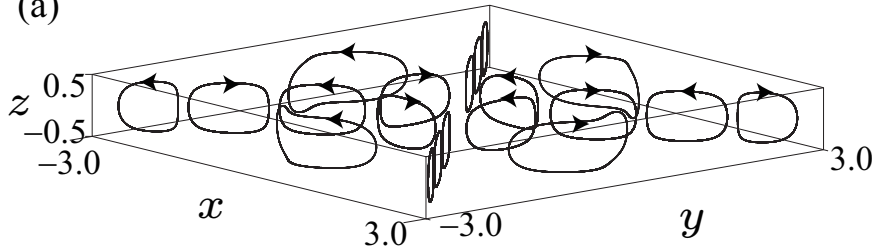

(b)

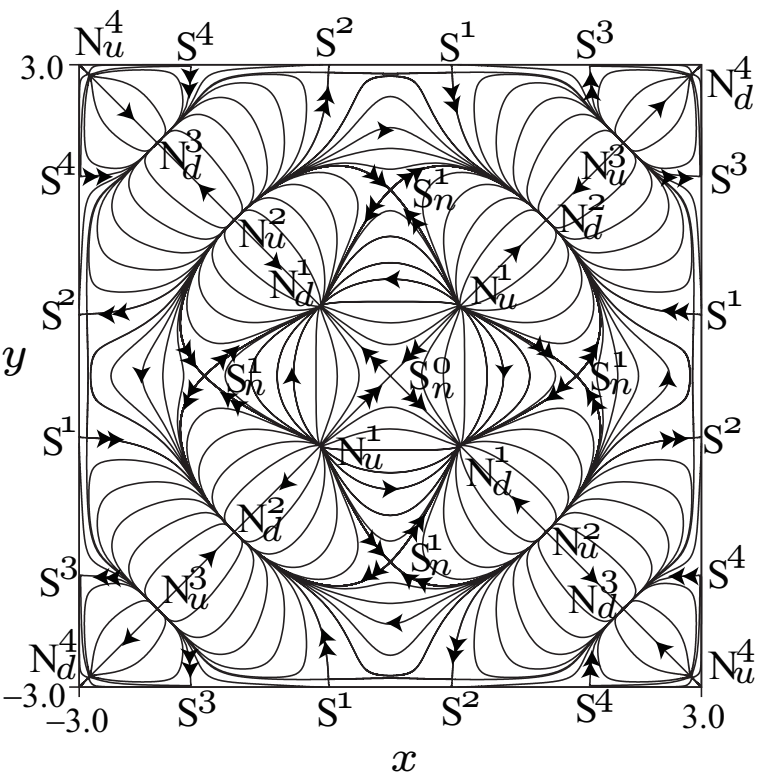

(c)

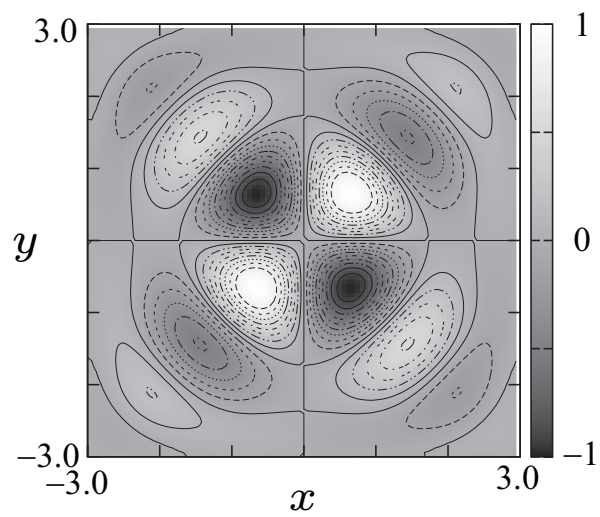

Figure 11. Characteristics of the flow of most unstable mode for $A=6$. (a) Trajectories of several fluid particles. (b) Upper-wall flow pattern. $\mathrm{N}_{u}^{j}$ and $\mathrm{N}_{d}^{j}(j=$ $1, \ldots, 4)$ denote upward and downward nodes of near-wall velocity field, respectively, $\mathrm{S}^{j}(j=1, \ldots, 4)$ denote upward or downward saddles, and $\mathrm{S}_{n}^{0}$ and $\mathrm{S}_{n}^{1}$ denote neutral saddles. (c) Distribution of $w$ on the horizontal plane $z=0$. Its value is normalized so that largest $|w|$ is 1 . 
fluid particles starting from one of the vertical planes $y= \pm x$ is restricted to this plane. However, four central convection cells and four convention cells adjacent to them do not extend to side walls, unlike the case of $A=4$. A horizontal scale of convection cells can be estimated from the upper-wall flow pattern shown in Fig. 11(b). The distances of four equilibrium points $\mathrm{S}_{n}^{0}, \mathrm{~N}_{u}^{1}, \mathrm{~N}_{d}^{2}$ and $\mathrm{N}_{u}^{3}$ on the diagonal line $y=x$ are $0.953,1.174$ and 1.050. Their average value 1.059, which can be considered as a typical width-to-height ratio of convection cells, is only slightly larger than the ratio 1.008 of convection cells of most unstable mode for an infinitely extended fluid layer obtained in the preceding studies. Although it is reasonable to expect that such an average value tends to 1.008 as $A$ increases to infinity, this expectation is not yet confirmed because the computation of most unstable mode for large $A$ with high accuracy is not easy.

Of the $\left|D_{w}\right|$ 's at equilibrium points of near-wall velocity field shown in Fig. 11(b), $\left|D_{w}\right|$ at nodes $\mathrm{N}_{d}^{1}$ and $\mathrm{N}_{u}^{1}$ close to the center is the largest. This $\left|D_{w}\right|$ is about twice as large as the $\left|D_{w}\right|$ at neighboring nodes $\mathrm{N}_{d}^{2}$ and $\mathrm{N}_{u}^{2}$, and is more than 6 times as large as $\left|D_{w}\right|$ 's at the remaining equilibrium points. Therefore, the strongest and the second strongest vertical flows are expected in the vertical cylindrical regions around $\mathrm{N}_{d}^{1}$ and $\mathrm{N}_{u}^{1}$ and around $\mathrm{N}_{d}^{2}$ and $\mathrm{N}_{u}^{2}$, respectively. These vertical flows are confirmed by the distribution of $w$ on the plane $z=0$ shown in Fig. 11(c). Although Stork and Müller (1972) observed for $A=6$ disturbances of the cell shape from that obtained by Davis (1967) under the assumption of finite roll, the detailed comparison with our results is impossible because no figure of experimental flow pattern was shown in their paper.

\section{Discussion and Conclusions}

Onset of thermal convection of a fluid in a rectangular cavity of aspect ratios $A_{x}$ and $A_{y}$ is examined under the assumption that its all walls are rigid and of perfect thermal conductance exposed to a vertically linear temperature field. For $A_{x}$ and $A_{y}$ smaller than 6, critical Rayleigh number $R_{c}$ and the flow patterns of most unstable modes are computed numerically by a Galerkin spectral method of high accuracy. The symmetry of most unstable mode is expressed as $\hat{\theta}\left(s_{x}, s_{y}, s_{z}\right)$, where $s_{x}$ (or $\left.s_{y}, s_{z}\right)=e[o]$ implies that temperature deviation $\hat{\theta}$ is an even [odd] function of $x$ (or $y, z$ ). Characteristics of flow patterns are examined by using upper-wall flow patterns based on near-wall velocity fields and distributions of vertical velocity on the central horizontal plane as well as trajectories of many fluid particles. It is found that the equilibrium points of near-wall velocity fields and streamlines connecting a saddle and an equilibrium point in the upper-wall flow patterns are important in the examination of geometrical structure of convection cells. These streamlines are upper ends of boundary surfaces of convection cells.

The following results on the dependence of $R_{c}$ and most unstable modes on aspect ratios are obtained: If condition $1 \leq A_{x} \ll A_{y}$ is satisfied, the flow pattern of most unstable mode in the region not close to side walls is a linear arrangement of several convection cells whose axes of circulation are horizontal and parallel to shorter side walls, 
consistently with the results obtained in preceding studies. As $A_{y}$ increases, $\hat{\theta}(e, e, e)$ and $\hat{\theta}(e, o, e)$ modes are alternately obtained as the most unstable mode, corresponding to the increase in the number of convection cells. There is rough consistency between our numerical results and experimental results by Stork and Müller (1972) on the $A_{y}$ 's at which the number of convection cells changes. The width-to-height ratio of convection cells is a little smaller than 1 for most $A_{y}$, and is close to the ratio for an infinite channel of square cross section if $A_{x}=1$ and $A_{y}$ is large. Moreover, the axes of circulation in convection cells near shorter side walls are strongly curved. It is also found that the validity of assumption of finite roll used by Davis (1967) is limited even if $A_{y}$ is much larger than $A_{x}$. As $A_{y}$ increases, $R_{c}$ for $A_{x}=1$ decreases slowly and asymptotes to the $R_{c}$ for an infinite channel of square cross section.

If $A_{x}$ [or $\left.A_{y}\right]$ is decreased to zero with $A_{y}\left[A_{x}\right]$ fixed to a value, we observe frequent changes of most unstable mode between $\hat{\theta}(e, e, e)$ and $\hat{\theta}(e, o, e)[\hat{\theta}(o, e, e)]$ modes. These changes occur more frequently for smaller $A_{x}\left[A_{y}\right]$. The increase in $R_{c}$ when either or both of $A_{x}$ and $A_{y}$ approach zero is quite rapid.

In the examination of most unstable mode for $A_{x} \leq 6$ and $A_{y} \leq 6$, we find that $\hat{\theta}(o, o, e)$ mode is the most unstable if both $A_{x}$ and $A_{y}$ are around 4 or around 5.5. As far as the authors know, the observation of this symmetry mode as the most unstable mode was not reported in the preceding studies for the boundary condition considered in the present study. The existence of two $\left(A_{x}, A_{y}\right)$ regions where $\hat{\theta}(o, o, e)$ mode is the most unstable yields 11 sets of aspect ratios $\left(A_{x}, A_{y}\right)$ for which critical Rayleigh numbers for three symmetry modes are the same. Moreover, the $R_{c}$ for a few aspect ratios is found to be roughly consistent with the $R_{c}$ in the preceding experimental studies.

In the detailed examination of $R_{c}$ and flow patterns of most unstable mode for a cavity of square base with $A_{x}=A_{y}=A \leq 6$, we obtain the following results: $R_{c}=6797.738$ for a cubic cavity of $A=1$ agrees well with the values obtained in the preceding studies. Moreover, $R_{c}$ decreases as $A$ increases, and $R_{c}=1761.788$ for $A=6$ is slightly larger than the $R_{c}$ for a fluid between two parallel walls of infinite extension.

For $A$ satisfying $A \leq 1.312,2.968 \leq A \leq 3.524$ or $4.418 \leq A \leq 5.023, \hat{\theta}(e, o, e)$ [and $\hat{\theta}(o, e, e)]$ mode is the most unstable. For $A=1$, the flow pattern of $\hat{\theta}(e, o, e)$ mode in the central region of cavity is a roll whose axis of circulation is horizontal and parallel to side walls $y= \pm 0.5$. The region within a cavity is composed of a main convection cell corresponding to this roll pattern and wall regions where fluid particles move along closed orbits that are always close to side walls $y= \pm 0.5$. Moreover, within the main convection cell, the motion of fluid particles is quasi two-dimensional unless they pass through the region close to the boundary surfaces of this cell. As $A$ increases from 1 to 1.312 , the volume ratios of main convection cell and the region of quasi two-dimensional motion decrease.

The flow patterns of $\hat{\theta}(e, o, e)$ mode for $A=3$ and 5 , within the second and third ranges of $A$ mentioned above, are characterized by strong vertical flows in the vertical cylindrical regions around two nodes that are close to the center and are on the symmetry line $x=0$ in the upper-wall flow pattern. For $A=3$, the region within a cavity 
is divided into a central convection cell, four convection cells adjacent to this cell, and small convection cells close to side walls. The motion of fluid particles within the central convection cell is roughly the circulation around a horizontal axis parallel to side walls $y= \pm 1.5$, similarly to the motion within the main convection cell for $A=1$. Within the adjacent convection cells, it is roughly said that fluid particles move around a horizontal axis that is not parallel to any side walls. The flow pattern for $A=5$ is more complicated and is composed of more convection cells than the flow pattern for $A=3$. The region within a cavity is divided into 19 convection cells, and in each cell fluid particles move around a horizontal axis. Although some of the axes are straight, the remaining axes are a little convex outward. A central convection cell and four adjacent convection cells observed in the flow pattern do not extend to side walls, unlike the case of $A=3$.

For $1.312 \leq A \leq 2.968, \hat{\theta}(e, e, e)$ mode is the most unstable. In the flow pattern for $A=2$ included in this range, the region within a cavity is divided into four central convection cells and a few convection cells near side walls. In the central convection cells, trajectories of fluid particles reveal radial circulation with a strong vertical flow in the central part of cavity. This motion is consistent with the top-view image of fluid motion obtained in the experiments by Stork and Müller (1972) for $A=2$.

$\hat{\theta}(o, o, e)$ mode is the most unstable for $3.524 \leq A \leq 4.418$ and $5.023 \leq A \leq 6$. The flow patterns of this mode for $A=4$ and 6 within these ranges are characterized by strong vertical flows in the vertical cylindrical regions around four nodes that are close to the center and are on the diagonal lines $y= \pm x$ in the upper-wall flow pattern. This property is consistent with the top-view image of fluid motion obtained in the experiments by Stork and Müller (1972) for $A=4$. In the flow pattern for $A=4$, the region within a cavity is occupied by 12 convection cells composed of four central, four middle, and four corner convection cells. Central convection cells almost extend to side walls, and the motion of fluid particles within them is roughly the circulation around a horizontal axis parallel to side walls. Fluid particles within middle convection cells move around a horizontal axis that is not parallel to side walls. In the flow pattern for $A=6$, 20 convection cells are observed within the region of cavity. In each convection cell, fluid particles move around a straight or curved axis located on the central horizontal plane. Four central convection cells and four convention cells adjacent to them do not extend to side walls, unlike the case of $A=4$. A typical width-to-height ratio of convection cells obtained from the flow pattern is only slightly larger than the ratio of the cells of most unstable mode for an infinitely extended fluid layer.

As found from the above results for a cavity of square base, the flow pattern becomes more complicated and the number of convection cells increases as $A$ increases. The motion of fluid particles in each cell is roughly the circulation along closed curves around an axis that is on the central horizontal plane but is not necessarily parallel to side walls. Moreover, the motion of fluid particles near side walls can be much different from that in the central part of cavity.

In his study under the assumption of finite roll, Davis (1967) showed that if one of the aspect ratios is smaller than 1, narrower cells of width smaller than its height 
are obtained as the most unstable mode. Although similar results are obtained in our study without this assumption, the details of flow patterns of most unstable mode for such aspect ratios as well as theoretical explanation of them will be reported in another paper in the near future.

Since there are many preceding studies related to the dependences of $R_{c}$ and the flow pattern of most unstable mode on the aspect ratios of a rectangular cavity with different boundary conditions, it seems interesting to compare the results in some of these studies with the present results. Edwards (1988) numerically investigated the $R_{c}$ and the flow pattern of most unstable mode for a rectangular cavity of insulating side walls with the same other boundary conditions as those in the present study. In the examination for $A_{x}$ and $A_{y}$ between 1 and 12, he found that $\hat{\theta}(o, o, e)$ mode is not the most unstable, although the regions of $\left(A_{x}, A_{y}\right)$ where $\hat{\theta}(e, o, e), \hat{\theta}(o, e, e)$, and $\hat{\theta}(e, e, e)$ modes are the most unstable obtained in his and our studies are similar except for $A_{x}$ and $A_{y}$ around $A_{x}=A_{y}=4$ or 5.5. Moreover, the flow pattern of most unstable $\hat{\theta}(e, e, e)$ mode for $A_{x}=A_{y}=4$ shown in Fig. 7(a) of his paper is associated with a strong vertical flow in the central part of cavity, unlike the flow pattern of most unstable $\hat{\theta}(o, o, e)$ mode for the same $A_{x}$ and $A_{y}$ obtained in the present study (shown in Fig. 9). Although these differences in the most unstable mode and its flow pattern suggest the non-negligible influence of boundary conditions on side walls even for aspect ratios larger than 4 , it is difficult to find the reason for the appearance of $\hat{\theta}(o, o, e)$ mode as the most unstable mode in the present study. Hirschberg and Knobloch (1997) theoretically investigated the dependence of onset of thermal convection on boundary conditions at side walls for a two-dimensional rectangular cavity in the limit of large aspect ratio $A_{x}$. Under the assumptions of insulating side walls and stress-free top and bottom walls, they showed that the shape of neutral curve depends on the generalized boundary condition of velocity field at side walls that includes non-slip (Dirichlet type) and stress-free (Neumann type) boundary conditions as special cases, and that $R_{c}$ varies by $O\left(A_{x}^{-3}\right)$ with the variation of this boundary condition for large $A_{x}$. Therefore, there may be a possibility that the appearance of most unstable $\hat{\theta}(o, o, e)$ mode for large $A_{x}$ and $A_{y}$ only in the present study of Dirichlet-type thermal boundary condition at side walls is explained on the basis of a similar analysis.

Gelfgat (1999) numerically investigated $R_{c}$ and the flow pattern of most unstable mode for a rectangular cavity with insulating and rigid side walls, a rigid bottom wall of constant temperature, and a stress-free top wall satisfying a mixed-type thermal condition specified by the Biot number $B_{i}$. He examined the case of a cavity of a square base $\left(A_{x}=A_{y}=A\right)$. The symmetry of each mode in his study is expressed as EE, EO, $\mathrm{OE}$ or OO, which is the same as the symmetry of $\hat{\theta}$ in the $x$ and $y$ directions in the present study (this mode cannot have the even or odd symmetry in the vertical direction because of the boundary conditions imposed at the top and bottom walls.). In his examination of $R_{c}$ and the most unstable mode for $1 \leq A \leq 8$ and $B i=1$, he found that the $\mathrm{OO}$ symmetry mode can be the most unstable for $4.98 \leq A \leq 5.38$. Therefore, the mode of the same symmetry in the horizontal directions as $\hat{\theta}(o, o, e)$ mode was observed also in 
this study with different boundary conditions at side and top walls. The flow pattern of most unstable $\mathrm{OO}$ mode shown in Fig. 8 of his paper for $A=5$ is similar to Fig. 9 for $A=4$ in the present paper in that there are four regions of strong vertical flows near vertical diagonal planes of the cavity, but is different in that his flow pattern is not invariant under the reflection with respect to vertical diagonal planes of $y= \pm x$, as found from Fig. 8(d) of his paper and Fig. 9(d) of the present paper. This difference and the discrepancy between the regions of $A$ where OO symmetry mode or $\hat{\theta}(o, o, e)$ mode is the most unstable are caused by the difference in boundary conditions. Dauby and Lebon (1996) numerically examined the onset of Marangoni convection for several aspect ratios of a rectangular cavity with insulating side walls and an upper surface satisfying slip and insulating boundary conditions. In the study of most unstable modes that appear at critical Marangoni numbers for $A_{x}$ and $A_{y}$ between 1 and 9 , they found that $\hat{\theta}(o, o, e)$ mode can be the most unstable for $\left(A_{x}, A_{y}\right)$ within two regions around $A_{x}=A_{y}=6$ and 9 , but that this mode is not the most unstable for $\left(A_{x}, A_{y}\right)$ around $A_{x}=A_{y}=4$. This result gives another example of the most unstable $\hat{\theta}(o, o, e)$ mode for some aspect ratios of a rectangular cavity.

There are also a few preceding studies on the steady solutions to nonlinear governing equations of thermal convection in a rectangular cavity. It seems interesting to compare the flow patterns of most unstable mode in the present study with those of the steady solutions obtained in some of these studies for Rayleigh number $R$ just above $R_{c}$. Sezai and Mohamad (2000) numerically investigated the flow pattern of thermal convection in a rectangular cavity of $A_{x}=A_{y}=4$ for several $R$ larger than $R_{c}$ using two kinds of thermal boundary conditions at side walls as well as the non-slip boundary condition at all the walls. The pattern of a nonlinear steady flow for the same boundary conditions as those in the present study calculated for $R=2000$ is shown in Figs. 10(a) and (b) and 11 of their paper. Because a strong vertical flow is observed at the center of the cavity in these figures, this flow pattern is not close to the flow pattern of most unstable $\hat{\theta}(o, o, e)$ mode for the same $A_{x}$ and $A_{y}$ and for $R=R_{c}=1838.152$ obtained in the present study, but is closer to $\hat{\theta}(e, e, e)$ mode that is not the most unstable. This problem may be resolved by the bifurcation analysis of nonlinear steady solutions for $R$ not far from $R_{c}$.

Trajectories of fluid particles for any $A_{x}$ and $A_{y}$ used in the present study are closed orbits (as shown in many figures of the present paper), stationary points, or heteroclinic orbits connecting equilibrium points of near-wall velocity fields or velocity fields on symmetry planes derived from $\hat{\boldsymbol{u}}$. Therefore, although chaotic trajectories are generally possible in three-dimensional steady flows, such trajectories are not observed for velocity field $\hat{\boldsymbol{u}}$. Arter $(1983,1985)$ examined the motion of fluid particles in steady three-dimensional flows of thermal convection in a rectangular cavity with boundary conditions that are different from ours in that all the walls are stress-free and side walls are insulating. He proved that trajectories in the velocity field of most unstable mode are always either periodic orbits or stationary points because of the existence of two constants of motion, and showed that trajectories can be chaotic if a steady velocity field approximately satisfying nonlinear governing equations of thermal convection when 
$R$ is slightly larger than $R_{c}$ is used instead of that of most unstable mode. Chernikov and Schmidt (1992) also examined regular and chaotic trajectories of fluid particles in these velocity fields. Although the non-existence of chaotic trajectories in the present study may be also attributed to the existence of constants of motion, they are not found yet. Chaotic or complicated trajectories in steady three-dimensional flows were also reported in numerical studies (Simó et al (2010) and Ozoe et al (1976)) and an experimental study (Ozoe et al (1979)) on thermal convection for $R$ larger than $R_{c}$. Therefore, also in the thermal convection system considered in the present study, chaotic trajectories may be possible if the velocity field satisfying nonlinear governing equations for $R$ larger than $R_{c}$ in place of the velocity field of most unstable mode is used. This possibility will be examined in the future study.

Acknowledgement This work is partly supported by the Grants-in-Aid (No.25390154) for Scientific Research of the Ministry of Education, Culture, Sports, Science and Technology.

\section{References}

Arter, W. 1983. Ergodic stream-lines in steady convection, Phys. Lett., 97A, pp.171-174.

Arter, W. 1985. Nonlinear Rayleigh-Bénard convection with square planform, J. Fluid Mech., 152, pp.391-418.

Bousset, F., Lyubimov, D. V. and Sedel'nikov, G. A. 2008. Three-dimensional convection regimes in a cubical cavity, Fluid Dyn., 43, pp.1-8.

Catton, I. 1970. Convection in a closed rectangular region: the onset of motion, ASME J. Heat Transfer, pp.186-188.

Chernikov, A. A. and Schmidt, G. 1992. Chaotic streamlines in convective cells, Phys. Lett. A, 169, pp.51-56.

Daniels, P. G. and Ong, C. F. 1990. Linear stability of convection in a rigid channel uniformly heated from below, Int. J. Heat Mass Transfer, 33, pp.55-60.

Dauby, P. C. and Lebon, G. 1996. Bénard-Marangoni instability in rigid rectangular containers, J. Fluid Mech., 329, pp.25-64.

Davies-Jones, R. P. 1970. Thermal convection in an infinite channel with no-slip sidewalls, J. Fluid Mech., 44, pp.695-704.

Davis, S. H. 1967. Convection in a box: linear theory, J. Fluid Mech., 30, pp.465478. 
Délery, J. M. 2001. Robert Legendre and Henri Werlé: Toward the elucidation of three-dimensional separation, Annu. Rev. Fluid Mech., 33, pp.129-154.

Edwards, B. F. 1988. Crossed rolls at onset of convection in a rigid box, J. Fluid Mech., 191, pp.583-597.

Gelfgat, A. Y. 1999. Different modes of Rayleigh-Bénard instability in two- and three-dimensional rectangular enclosures. J. Comput. Phys., 156, pp.300-324.

Gershuni, G. Z. and Zhukhovitskii, E. M. 1972. "Convective Stability of Incompressible Fluids," (Nauka, Moscow, English Translation: Keter Publishing House, Jerusalem, 1976) Chapter IV, $§ 18$.

Hirschberg, P. and Knobloch, E. 1997. Mode interactions in large aspect ratio convection, J. Nonl. Sci., 7, pp.537-556.

Kessler, R. 1987. Nonlinear transition in three-dimensional convection, J. Fluid Mech., 174, pp.357-379.

Kirchartz, K. R. and Oertel Jr, H. 1988. Three-dimensional thermal cellular convection in rectangular boxes, J. Fluid Mech., 192, pp.249-286.

Koschmieder, E. L. 1966. On convection on a uniformly heated plane, Beitr. Phys. Atmos., 39, pp.1-11.

Koschmieder, E. L. 1993. "Bénard Cells and Taylor Vortices," (Cambridge University Press) Section 5.2.

Leong, W. H., Hollands, K. G. T. and Brunger, A. P. 1998. On a physicallyrealizable benchmark problem in internal natural convection, Int. J. Heat Mass Transfer, 41, pp.3817-3828.

Mizushima, J. and Matsuda, O. 1996. (in Japanese) Res. Inst. Appl. Math., Kyoto Univ. Kokyuroku, 970, pp.1-9.

Mizushima, J. and Matsuda, O. 1997. Onset of 3D thermal convection in a cubic cavity, J. Phys. Soc. Jpn., 66, pp.2337-2341.

Ozoe, H., Sato, N. and Churchill, S. 1979. Experimental confirmation of the three-dimensional helical streaklines previously computed for natural convection in inclined rectangular enclosures, Int. Chem. Eng., 19, pp.454-462.

Ozoe, H., Yamamoto, K., Churchill, S. W. and Sayama, H. 1976. Threedimensional, numerical analysis of laminar natural convection in a confined fluid heated from below, ASME J. Heat Transfer, 98C, pp.202-207.

Puigjaner, D., Herrero, J., Giralt, F. and Simó, C. 2004. Stability analysis of the flow in a cubical cavity heated from below, Phys. Fluids, 16, pp.3639-3655. 
Puigjaner, D., Herrero, J., Simó, C. and Giralt, F. 2008. Bifurcation analysis of steady Rayleigh-Bénard convection in a cubical cavity with conducting sidewalls, J. Fluid Mech., 598, pp.393-427.

Sezai, I. and Mohamad, A. A. 2000. Natural convection in a rectangular cavity heated from below and cooled from top as well as the sides, Phys. Fluids, 12, pp.432-443.

Sherman, M. and Ostrach, S. 1966. On the principle of exchange of stabilities for the magnetohydrodynamic thermal stability problem in completely confined fluids, J. Fluid Mech., 24, pp.661-671.

Simó, C., Puigjaner, D., Herrero, J. and Giralt, F. 2010. Dynamics of particle trajectories in a Rayleigh-Bénard problem, Commun. Nonl. Sci. Numer. Simulat., 15, pp.24-39.

Stork, K. and Müller, U. 1972. Convection in boxes: experiments, J. Fluid Mech., 54, pp.599-611.

Surana, A., Grunberg, O. and Haller, G. 2006. Exact theory of three-dimensional flow separation. Part 1. Steady separation, J. Fluid Mech., 564, pp.57-103.

Surana, A., Jacobs, G. B. and Haller, G. 2007. Extraction of separation and attachment surfaces from three-dimensional steady shear flows, AIAA J., 45, pp.1290-1302. 